\title{
A Stereoselective Three-Component Reaction: KAl(SO4)2.12H2O an Efficient and Reusable Catalyst for the One-Pot Synthesis of cis-Isoquinolonic Acids
}

\section{Javad Azizian,* Ali A. Mohammadi, Ali R. Karimi, Mohammad R. Mohammadizadeh}

Department of Chemistry, Faculty of Science, Shahid Beheshti University, P. O. Box 19395-4716, Tehran, Iran, E-mail: j-azizian@cc.sbu.ac.ir

\section{SUPPORTING INFORMATION}

Detailed list of CONTENTS of the supporting information

\begin{tabular}{|c|c|c|c|}
\hline No & List of contents & Supporting info. No. & page \\
\hline 01 & Title, author's name and address & - & S1 \\
\hline 02 & Experimental Section & - & S4 \\
\hline 03 & $\begin{array}{l}\text { General Procedure for the Preparation of } \\
\text { cis-Isoquinolonic Acids }\end{array}$ & - & S4 \\
\hline 04 & General Procedure for Recovered Catalyst & - & S4 \\
\hline 05 & $\begin{array}{l}\text { Spectral data for Products and } \\
\text { intermediates }\end{array}$ & - & S4 \\
\hline 06 & IR 5a & S01 & S10 \\
\hline 07 & ${ }^{1} \mathrm{H}$ NMR of $\mathbf{5 a}$ & $\mathrm{S} 02$ & S11 \\
\hline 08 & IR 5b & S03 & S12 \\
\hline 09 & ${ }^{1} \mathrm{H}$ NMR of $\mathbf{5 b}$ & S04 & S13 \\
\hline 10 & ${ }^{13} \mathrm{C}$ NMR of $\mathbf{5 b}$ & S05 & S14 \\
\hline 11 & IR of $\mathbf{5 b}, \mathbf{4 b}$ & S06 & S15 \\
\hline 12 & ${ }^{1} \mathrm{H}$ NMR of $\mathbf{5 b}, \mathbf{4 b}$ & S07 & S16 \\
\hline 13 & ${ }^{1} \mathrm{H}$ NMR of $\mathbf{5 b} \mathbf{b} \mathbf{4 b}$ (expanded) & S08 & S17 \\
\hline
\end{tabular}




\begin{tabular}{|c|c|c|c|}
\hline 14 & ${ }^{1}$ H NMR of $\mathbf{5 b}, \mathbf{4 b}$ (expanded) & S09 & S18 \\
\hline 15 & ${ }^{13} \mathrm{C}$ NMR of $\mathbf{5 b}, \mathbf{4 b}$ & S10 & S19 \\
\hline 16 & ${ }^{13} \mathrm{C}$ NMR Mixture of $\mathbf{5 b}, \mathbf{4 b}$ (expanded) & S11 & S20 \\
\hline 17 & IR 4b & S12 & S21 \\
\hline 18 & ${ }^{1} \mathrm{H}$ NMR of $\mathbf{4 b}$ & S13 & S22 \\
\hline 19 & ${ }^{1}$ H NMR 4b(expanded) & S14 & S23 \\
\hline 20 & 夏H NMR 4b(expanded) & S15 & S24 \\
\hline 21 & ${ }^{13} \mathrm{C} \mathrm{NMR}$ of $\mathbf{4 b}$ & S16 & S25 \\
\hline 22 & ${ }^{1} \mathrm{H} N M R$ of $\mathbf{4 c}$ & S17 & S26 \\
\hline 23 & ${ }^{\mathrm{T}} \mathrm{H}$ NMR 4c(expanded) & S18 & S27 \\
\hline 24 & ${ }^{13} \mathrm{C}$ NMR of $4 c$ & S19 & S28 \\
\hline 25 & ${ }^{1}$ H NMR of 4d & S20 & S29 \\
\hline 26 & ${ }^{13} \mathrm{C}$ NMR of $\mathbf{4 d}$ & S21 & S30 \\
\hline 27 & ${ }^{13}$ C NMR 4d(expanded) & S22 & S31 \\
\hline 28 & ${ }^{1} \mathrm{H}$ NMR of $\mathbf{4 e}$ & S23 & S32 \\
\hline 29 & ${ }^{1}$ H NMR 4e(expanded) & S24 & S33 \\
\hline 30 & ${ }^{13} \mathrm{C}$ NMR of $4 \mathbf{e}$ & S25 & S34 \\
\hline 31 & ${ }^{13} \mathrm{C}$ NMR 4e(expanded) & S26 & S35 \\
\hline 32 & ${ }^{1} \mathrm{H}$ NMR of $\mathbf{4 f}$ & S27 & S36 \\
\hline 33 & ${ }^{13} \mathrm{C}$ NMR of $\mathbf{4 f}$ & S28 & S37 \\
\hline 34 & ${ }^{1} \mathrm{H}$ NMR of $\mathbf{4 g}$ & S29 & S38 \\
\hline 35 & ${ }^{13} \mathrm{C}$ NMR of $\mathbf{4 g}$ & S30 & S39 \\
\hline 36 & ${ }^{1} \mathrm{H}$ NMR of $\mathbf{4 h}$ & S31 & S40 \\
\hline 37 & ${ }^{1}$ H NMR 4h(expanded) & S32 & S41 \\
\hline 38 & ${ }^{13} \mathrm{C}$ NMR of $\mathbf{4 h}$ & S33 & S42 \\
\hline 39 & ${ }^{1} \mathrm{H}$ NMR of $\mathbf{4 i}$ & S34 & S43 \\
\hline 40 & 'H NMR 4i(expanded) & S35 & S44 \\
\hline 41 & ${ }^{13} \mathrm{C} \mathrm{NMR}$ of $\mathbf{4 i}$ & S36 & S45 \\
\hline 42 & ${ }^{1}$ H NMR of $4 \mathbf{l}$ & S37 & S46 \\
\hline
\end{tabular}




\begin{tabular}{|c|c|c|c|}
\hline 43 & ${ }^{1}$ H NMR 4l(expanded) & S38 & S47 \\
\hline 44 & ${ }^{13}$ C NMR of 4l & S39 & S48 \\
\hline 45 & ${ }^{1}$ H NMR of 4m & S40 & S49 \\
\hline 46 & ${ }^{1}$ H NMR 4m(expanded) & S41 & S50 \\
\hline 47 & ${ }^{13}$ C NMR of $\mathbf{4 m}$ & S42 & S51 \\
\hline 48 & ${ }^{13}$ C NMR 4m(expanded) & S43 & S52 \\
\hline
\end{tabular}




\section{Experimental Section:}

Melting points were measured on the Electrothermal 9100 apparatus and are uncorrected. IR spectra were

measured on a Shimadzu IR-470 Spectrophotometer. ${ }^{1} \mathrm{H}$ NMR and ${ }^{13} \mathrm{C}$ NMR spectra were determined on a bruker 500 DRX AVNCE instrument at 500 and $125 \mathrm{MHz}$, respectively. Ms spectra were recorded on a Shimadzu QP 1100EX mass spectrometer operating at an ionization potential of $70 \mathrm{eV}$. Elemental analyses were performed using a Heracus CHN-O-Rapid analyzer.

\section{General Procedure for the Preparation of $c$ is-Isoquinolonic Acids:}

A mixture of homophthalic anhydride $(162 \mathrm{mg}, 1 \mathrm{mmol})$, aldehydes $(1.2 \mathrm{mmol})$, amines $(1 \mathrm{mmol})$, alum $(0.24 \mathrm{~g}, 0.5 \mathrm{mmol})$ and acetonitrile $(8-10 \mathrm{~mL})$ in a $25 \mathrm{~mL}$ flask was stirred at $\mathrm{rt}$ for the time period as indicated in Table 2. After completion of the reaction (monitored by TLC, ethyl acetate/n-hexane 1/1), the solvent was evaporated under reduced pressure, then water $(25 \mathrm{~mL})$ was add to the reaction mixture and the resulting solid was separated by filtration. The crude product was washed with chloroform and ether to afford pure cis-isoquinolonic acids in $82-94 \%$ yields.

\section{General Procedure for Recovered Catalyst:}

The catalyst in the aqueous phase can be recovered by removing the water under vacuum then washed with acetone and drying at room temperature.

\section{Spectral data for Products and intermediates:}

5a: White powder, yield $(245 \mathrm{mg}) 96 \%, \mathrm{mp} 188-90{ }^{\circ} \mathrm{C}, \mathrm{IR}(\mathrm{KBr}),\left(v_{\max } / \mathrm{cm}^{-1}\right): 3295,3045,2860,1700,1665$.

${ }^{1} \mathrm{H}$ NMR $\left(\mathrm{CD}_{3} \mathrm{SOCD}_{3}\right) \delta_{\mathrm{H}}: 4.07\left(\mathrm{~s}, 2 \mathrm{H}, \mathrm{CH}_{2}\right), 6.99(\mathrm{~m}, 1 \mathrm{H}), 7.26-7.35(\mathrm{~m}, 4 \mathrm{H}), 7.49-7.56(\mathrm{~m}, 3 \mathrm{H}), 7.83(\mathrm{~d}$, 
1H, J=6.7 Hz), 10.04 (s, 1H), 12.70 (broad, 1H). MS (m/z, \%): $255\left(\mathrm{M}^{+}, 35\right), 237(35), 209$ (25), 181 (25), 135 (55), 118 (50), 93 (100), 90 (45), 77 (50), 63 (25), 51 (20), 39 (30).

5b: White powder, yield $(280 \mathrm{mg}) 97 \%, \mathrm{mp} 196-8{ }^{\circ} \mathrm{C}, \mathrm{IR}(\mathrm{KBr}),\left(v_{\max } / \mathrm{cm}^{-1}\right): 3285,3030,2870,1702,1665$. ${ }^{1} \mathrm{H}$ NMR $\left(\mathrm{CD}_{3} \mathrm{SOCD}_{3}\right) \delta_{\mathrm{H}}: 4.08\left(\mathrm{~s}, 2 \mathrm{H}, \mathrm{CH}_{2}\right), 7.34-7.56(\mathrm{~m}, 7 \mathrm{H}), 7.87(\mathrm{~d}, 1 \mathrm{H}, J=9.00 \mathrm{~Hz}), 10.19(\mathrm{~s}, 1 \mathrm{H})$ $12.83(\mathrm{~s}, 1 \mathrm{H}) .{ }^{13} \mathrm{C} \mathrm{NMR}\left(\mathrm{CD}_{3} \mathrm{SOCD}_{3}\right) \delta_{\mathrm{C}}: 41.8,114.2,120.7,126.8,130.1,130.9,131.4,131.6,132.3$ 136.5, 138.7, 168.4, 169.2. MS (m/z,\%): $289\left(\mathrm{M}^{+}, 65\right), 271(50), 243(40), 215(35), 180(15), 135(50), 127$ (100), 118 (25), 90 (35), 77 (25), 63 (25), 44 (25).

5b and 4b: IR $(\mathrm{KBr}),\left(v_{\max } / \mathrm{cm}^{-1}\right): 3285,3050,1722,1700,1632 .{ }^{1} \mathrm{H} \mathrm{NMR}\left(\mathrm{CD}_{3} \mathrm{SOCD}_{3}\right) \delta_{\mathrm{H}}: 4.08(\mathrm{~s}, 2 \mathrm{H}$, $\left.\mathrm{CH}_{2}\right), 4.86\left(\mathrm{~d}, 1 \mathrm{H}, J=5.2 \mathrm{~Hz}, \mathrm{H}_{3}\right), 5.52\left(\mathrm{~d}, 1 \mathrm{H}, J=5.2 \mathrm{~Hz}, \mathrm{H}_{4}\right), 7.04-7.60(\mathrm{~m}, 14 \mathrm{H}), 7.87(\mathrm{~d}, 1 \mathrm{H}, J=7.5 \mathrm{~Hz})$, $8.06(\mathrm{~d}, 1 \mathrm{H}, J=7.6 \mathrm{~Hz}), 10.19(\mathrm{~s}, 1 \mathrm{H}), 12.90($ broad, $2 \mathrm{H}) .{ }^{13} \mathrm{C} \mathrm{NMR}\left(\mathrm{CD}_{3} \mathrm{SOCD}_{3}\right) \delta_{\mathrm{C}}: 42.3,49.6,64.5$, $120.9,126.8,127.3,128.2,128.2,128.3,128.4,128.5,128.6,128.9,129.3,129.7,130.7,131.3,132.8$ $132.9,134.9,137.5,140.8,163.5,168.9,169.7,170.9$.

Cis-1-Oxo-2,3-diphenyl-1,2,3,4-tetrahydroisoquinoline-4-carboxlic acid (4a): White powder, yield (302 $\mathrm{mg}) 88 \%, \mathrm{mp} 201-3{ }^{\circ} \mathrm{C}, \mathrm{IR}(\mathrm{KBr}),\left(v_{\max } / \mathrm{cm}^{-1}\right): 3020,2915,1723,1633 .{ }^{1} \mathrm{H} \mathrm{NMR}\left(\mathrm{CD}_{3} \mathrm{SOCD}_{3}\right) \delta_{\mathrm{H}}: 4.94(\mathrm{~d}$, $\left.1 \mathrm{H}, J=6.9 \mathrm{~Hz}, \mathrm{H}_{3}\right), 5.49\left(\mathrm{~d}, 1 \mathrm{H}, J=6.9 \mathrm{~Hz}, \mathrm{H}_{4}\right), 7.01-7.30(\mathrm{~m}, 10 \mathrm{H}), 7.57-7.60(\mathrm{~m}, 3 \mathrm{H}), 8.07(\mathrm{~d}, 1 \mathrm{H}, J=8.7$ $\mathrm{Hz}), 12.04$ (broad, $1 \mathrm{H}) .{ }^{13} \mathrm{C} \mathrm{NMR}\left(\mathrm{CD}_{3} \mathrm{SOCD}_{3}\right) \delta_{\mathrm{C}}: 49.5,65.1,126.6,128.1,128.2,128.3,128.4,128.4$, 128.6, 129.4, 129.6, 132.6, 135.9, 136.1, 137.3, 139.5, 163.2, 171.1. MS (m/z, \%): $343\left(\mathrm{M}^{+}, 75\right), 298(30)$, 207 (25), 181 (100), 134 (45), 118 (35), 105 (35), 90 (25), 77 (80), 63 (35), 51 (50), 39 (35).Anal. Calcd for $\mathrm{C}_{22} \mathrm{H}_{17} \mathrm{NO}_{3}$ : C, 76.95; H, 4.99; N, 4.08. Found: C, 76.78; H, 4.90; N, 4.00.

cis-2-(4-Chlorophenyl)-1-oxo-3-phenyl-1,2,3,4-tetrahydroisoquinoline-4-carboxlic acid (4b): White powder, yield (332 mg) 90\%, mp 200-1 ${ }^{\circ} \mathrm{C}$, IR (KBr), $\left(v_{\max } / \mathrm{cm}^{-1}\right): 3070,2913,1723,1630 .{ }^{1} \mathrm{H}$ NMR $\left(\mathrm{CD}_{3} \mathrm{SOCD}_{3}\right) \delta_{\mathrm{H}}: 4.86\left(\mathrm{~d}, 1 \mathrm{H}, J=4.6 \mathrm{~Hz}, \mathrm{H}_{3}\right), 5.52\left(\mathrm{~d}, 1 \mathrm{H}, J=4.6 \mathrm{~Hz}, \mathrm{H}_{4}\right), 7.04-7.57(\mathrm{~m}, 12 \mathrm{H}), 8.06(\mathrm{~d}, 1 \mathrm{H}$, $J=7.3 \mathrm{~Hz}), 12.91$ (broad, $1 \mathrm{H}) .{ }^{13} \mathrm{C} \mathrm{NMR}\left(\mathrm{CD}_{3} \mathrm{SOCD}_{3}\right) \delta_{\mathrm{C}}: 49.6,64.5,128.1,128.2,128.3,128.4,128.5$, 
128.6, 129.0, 129.3, 129.7, 131.3, 132.9, 134.9, 137.5, 140.8, 163.5, 170.9. MS (m/z, \%): $377\left(\mathrm{M}^{+}, 30\right), 358$ (20), 333 (60), 250 (20), 214 (100), 178 (50), 138 (30), 118 (95), 111 (80), 90 (95), 75 (80), 63 (85), 51 (70), 39 (55). Anal. Calcd for $\mathrm{C}_{22} \mathrm{H}_{16} \mathrm{NO}_{3} \mathrm{Cl}$ : C, 69.94; H, 4.27; N, 3.71. Found: C, 69.87; H, 4.18; N, 3.60.

cis-1-Oxo-3-phenyl-2-p-tolyl-1,2,3,4-tetrahydroisoquinoline-4-carboxlic acid (4c): White powder, yield (325 mg) 91\%, mp 187-8 ${ }^{\circ} \mathrm{C}, \mathrm{IR}(\mathrm{KBr}),\left(v_{\max } / \mathrm{cm}^{-1}\right): 3010,2910,1723,1614 .{ }^{1} \mathrm{H} \mathrm{NMR}\left(\mathrm{CD}_{3} \mathrm{SOCD}_{3}\right) \delta_{\mathrm{H}}$ : $2.23\left(\mathrm{~s}, 3 \mathrm{H}, \mathrm{CH}_{3}\right), 4.93\left(\mathrm{~d}, 1 \mathrm{H}, J=5.7 \mathrm{~Hz}, \mathrm{H}_{3}\right), 5.43\left(\mathrm{~d}, 1 \mathrm{H}, J=5.7 \mathrm{~Hz}, \mathrm{H}_{4}\right), 6.99-7.15(\mathrm{~m}, 9 \mathrm{H}), 7.45-7.60(\mathrm{~m}$, $3 \mathrm{H}), 8.05(\mathrm{~d}, 1 \mathrm{H}, J=7.6 \mathrm{~Hz}), 12.90$ (broad, $1 \mathrm{H}) .{ }^{13} \mathrm{C} \mathrm{NMR}\left(\mathrm{CD}_{3} \mathrm{SOCD}_{3}\right) \delta_{\mathrm{C}}: 21.0,49.4,65.0,127.6,128.0$, $128.2,128.2,128.3,128.4,128.5,129.5,129.6,132.6,134.7,136.3,137.7,139.6,163.3,170.8 . \mathrm{MS}(\mathrm{m} / z$, \%): $357\left(\mathrm{M}^{+}, 30\right), 313$ (35), 236 (25), 208 (45), 195 (100), 178 (70), 134 (30), 118 (75), 91 (85), 77 (95), 63 (50), 51 (70), 39 (55). Anal. Calcd for $\mathrm{C}_{23} \mathrm{H}_{19} \mathrm{NO}_{3}$ : C, 77.29; H, 5.36; N, 3.92. Found: C, 77.17; H, 5.29; N, 3.85 .

cis-2-Benzyl-1-oxo-3-phenyl-1,2,3,4-tetrahydroisoquinoline-4-carboxlic acid (4d): White powder, yield (315 mg) 88\%, mp 179-80 ${ }^{\circ} \mathrm{C}$, IR ( $\left.\mathrm{KBr}\right),\left(v_{\max } / \mathrm{cm}^{-1}\right): 3025,2910,1742,1613 .{ }^{1} \mathrm{H} \mathrm{NMR}\left(\mathrm{CD}_{3} \mathrm{SOCD}_{3}\right) \delta_{\mathrm{H}}$ : $3.75\left(\mathrm{~d}, 1 \mathrm{H}, J=15.3 \mathrm{~Hz}, \mathrm{CH}_{2}\right), 4.69\left(\mathrm{~d}, 1 \mathrm{H}, J=6.0 \mathrm{~Hz}, \mathrm{H}_{3}\right), 4.96\left(\mathrm{~d}, 1 \mathrm{H}, J=6.0 \mathrm{~Hz}, \mathrm{H}_{4}\right), 5.31(\mathrm{~d}, 1 \mathrm{H}, J=15.3$ $\left.\mathrm{Hz}, \mathrm{CH}_{2}\right), 6.96-7.57(\mathrm{~m}, 13 \mathrm{H}), 8.08(\mathrm{~d}, 1 \mathrm{H}, J=7.5 \mathrm{~Hz}), 12.99$ (broad, $\left.1 \mathrm{H}\right) .{ }^{13} \mathrm{C} \mathrm{NMR}\left(\mathrm{CD}_{3} \mathrm{SOCD}_{3}\right) \delta_{\mathrm{C}}: 48.5$, 48.6, 61.1, 127.6, 127.9, 128.0, 128.1, 128.2, 128.5, 128.7, 129.0, 129.2, 132.5, 134.1, 137.7, 163.5, 170.7 . MS (m/z, \%): $358\left(\mathrm{M}^{+}, 25\right), 357$ (35), 269 (30), 207 (25), 136 (30), 118 (80), 116 (75), 91 (100), 77 (35), 62 (30), 51 (35), 39(30). Anal. Calcd for $\mathrm{C}_{23} \mathrm{H}_{19} \mathrm{NO}_{3}$ : C, 77.29; H, 5.36; N, 3.92. Found: C, 77.20; H, 5.27; N, 3.83 .

cis-2-Benzyl-3-(4-chlorophenyl)-1-oxo-1,2,3,4-tetrahydroisoquinoline-4-carboxlic acid (4e): White powder, yield $(340 \mathrm{mg}) 87 \%, \mathrm{mp} 180-1{ }^{\circ} \mathrm{C}$, IR $(\mathrm{KBr}),\left(v_{\max } / \mathrm{cm}^{-1}\right): 3050,2905,1737,1608 .{ }^{1} \mathrm{H}$ NMR $\left(\mathrm{CD}_{3} \mathrm{SOCD}_{3}\right) \delta_{\mathrm{H}}: 3.83\left(\mathrm{~d}, 1 \mathrm{H}, J=15.3 \mathrm{~Hz}, \mathrm{CH}_{2}\right), 4.70\left(\mathrm{~d}, 1 \mathrm{H}, J=6.2 \mathrm{~Hz}, \mathrm{H}_{3}\right), 4.99\left(\mathrm{~d}, 1 \mathrm{H}, J=6.2 \mathrm{~Hz}, \mathrm{H}_{4}\right), 5.24$ (d, $1 \mathrm{H}, J=15.3 \mathrm{~Hz}, \mathrm{CH}_{2}$ ), 6.94-7.55 (m, 13H), 8.07 (d, 1H, J=7.5 Hz), 13.02 (broad, $\left.1 \mathrm{H}\right) .{ }^{13} \mathrm{C}$ NMR 
$\left(\mathrm{CD}_{3} \mathrm{SOCD}_{3}\right) \delta_{\mathrm{C}}: 48.5,48.7,60.5,127.6,128.0,128.1,128.4,128.7,128.9,129.0,130.0,132.6,133.3$, 133.9, 136.2, 137.6, 163.4, 170.6. MS (m/z, \%): $391\left(\mathrm{M}^{+}, 15\right), 347$ (40), 242 (40), 207 (30), 178 (35), 118 (45), 106 (45), 91 (100), 65 (50), 51 (30), 39 (30). Anal. Calcd for $\mathrm{C}_{23} \mathrm{H}_{18} \mathrm{NO}_{3} \mathrm{Cl}: \mathrm{C}, 70.50 ; \mathrm{H}, 4.63 ; \mathrm{N}, 3.57$. Found: C, 70.43; H, 4.54; N, 3.51 .

cis-1-Oxo-2-phenylethyl-3-phenyl-1,2,3,4-tetrahydroisoquinoline-4-carboxlic acid (4f): White powder, yield $(312 \mathrm{mg}) 84 \%, \mathrm{mp} 168-70{ }^{\circ} \mathrm{C}, \mathrm{IR}(\mathrm{KBr}),\left(v_{\max } / \mathrm{cm}^{-1}\right): 3025,2910,1738,1614 .{ }^{1} \mathrm{H} \mathrm{NMR}\left(\mathrm{CD}_{3} \mathrm{SOCD}_{3}\right)$ $\delta_{\mathrm{H}}: 2.67\left(\mathrm{~m}, 1 \mathrm{H}, \mathrm{CH}_{2}\right), 2.86-2.92\left(\mathrm{~m}, 1 \mathrm{H}, \mathrm{CH}_{2}\right), 2.98-3.04\left(\mathrm{~m}, 1 \mathrm{H}, \mathrm{CH}_{2}\right), 4.01-4.06\left(\mathrm{~m}, 1 \mathrm{H}, \mathrm{CH}_{2}\right), 4.52(\mathrm{~d}$, $\left.1 \mathrm{H}, J=6.2 \mathrm{~Hz}, \mathrm{H}_{3}\right), 4.98\left(\mathrm{~d}, 1 \mathrm{H}, J=6.2 \mathrm{~Hz}, \mathrm{H}_{4}\right), 6.97(\mathrm{~d}, 2 \mathrm{H}, J=6.2 \mathrm{~Hz}), 7.16-7.19(\mathrm{~m}, 6 \mathrm{H}), 7.25-7.28(\mathrm{~m}$, $2 \mathrm{H}), 7.41-7.44(\mathrm{~m}, 1 \mathrm{H}), 7.49(\mathrm{~m}, 2 \mathrm{H}), 8.02(\mathrm{~d}, 1 \mathrm{H}, J=6.8 \mathrm{~Hz}), 12.91$ (broad, $1 \mathrm{H}) .{ }^{13} \mathrm{C} \mathrm{NMR}\left(\mathrm{CD}_{3} \mathrm{SOCD}_{3}\right)$ $\delta_{\mathrm{C}}: 34.0,48.1,48.4,62.0,126.7,127.7,127.8,128.2,128.3,128.6,128.7,128.9,129.0,129.4,132.3,134.0$, 137.7, 139.5, 163.1, 170.7. MS (m/z, \%): $371\left(\mathrm{M}^{+}, 10\right), 353$ (15), 327 (25), 280 (35), $236(80), 221$ (30), 207 (60), 174 (40), 146 (25), 118 (40), 91 (100), 92 (30), 65 (30), 51 (30), 44 (30), 39 (25). Anal. Calcd for $\mathrm{C}_{24} \mathrm{H}_{21} \mathrm{NO}_{3}: \mathrm{C}, 77.60 ; \mathrm{H}, 5.70 ; \mathrm{N}, 3.77$. Found: C, 77.53; H, 5.62; N, 3.68 .

cis-2-(2-1H-Benzoimidazolyl)-1-oxo-3-phenyl-1,2,3,4-tetrahydroisoquinoline-4-carboxlic acid (4g): White powder, yield $(325 \mathrm{mg}) 85 \%$, mp 222-4 dec. ${ }^{\circ} \mathrm{C}, \mathrm{IR}(\mathrm{KBr}),\left(v_{\max } / \mathrm{cm}^{-1}\right): 3060,2915,1700,1657 .{ }^{1} \mathrm{H}$ $\operatorname{NMR}\left(\mathrm{CD}_{3} \mathrm{SOCD}_{3}\right) \delta_{\mathrm{H}}: 4.61\left(\mathrm{~d}, 1 \mathrm{H}, J=6.5 \mathrm{~Hz}, \mathrm{H}_{3}\right), 5.96\left(\mathrm{~d}, 1 \mathrm{H}, J=6.5 \mathrm{~Hz}, \mathrm{H}_{4}\right), 7.29-7.43(\mathrm{~m}, 7 \mathrm{H}), 7.47-7.57$ (m, 3H), $7.65(\mathrm{~m}, 1 \mathrm{H}), 7.95(\mathrm{~d}, 1 \mathrm{H}, J=7.7 \mathrm{~Hz}), 8.03$ (d, 1H, $J=7.6 \mathrm{~Hz}), 12.53$ (broad, 1H). ${ }^{13} \mathrm{C}$ NMR $\left(\mathrm{CD}_{3} \mathrm{SOCD}_{3}\right) \delta_{\mathrm{C}}: 49.2,79.0,80.3,124.9,126.2,127.3,128.1,128.2,128.7,128.9,129 ., 129.1,129.2,129.8$ 130.2, 134.4, 134.8, 137.2, 137.9, 163.9, 171.5. MS (m/z, \%): $383\left(\mathrm{M}^{+}, 10\right), 368(20), 313(15), 250(35)$, 178 (35), 118 (100), 105 (60), 90 (95), 77 (75), 63 (55), 51 (65), 39 (45). Anal. Calcd for $\mathrm{C}_{23} \mathrm{H}_{17} \mathrm{~N}_{3} \mathrm{O}_{3}$ : C, 72.05; H, 4.47; N, 10.96. Found: C, 71.91; H, 4.40; N, 10.87.

cis-3-(4-Nitrophenyl)-1-oxo-2-p-tolyl-1,2,3,4-tetrahydroisoquinoline-4-carboxlic acid (4h): White powder, yield (346 mg) 86\%, mp 246-8 dec. ${ }^{\circ} \mathrm{C}$, IR (KBr), $\left(v_{\max } / \mathrm{cm}^{-1}\right): 3065,2915,1723,1630,1598 .{ }^{1} \mathrm{H}$ 
$\operatorname{NMR}\left(\mathrm{CD}_{3} \mathrm{SOCD}_{3}\right) \delta_{\mathrm{H}}: 2.23\left(\mathrm{~s}, 3 \mathrm{H}, \mathrm{CH}_{3}\right), 4.99\left(\mathrm{~d}, 1 \mathrm{H}, J=5.1 \mathrm{~Hz}, \mathrm{H}_{3}\right), 5.97\left(\mathrm{~d}, 1 \mathrm{H}, J=5.1 \mathrm{~Hz}, \mathrm{H}_{4}\right), 7.09(\mathrm{~m}$, 4H), $7.31(\mathrm{~d}, 2 \mathrm{H}, J=8.2), 7.49-7.61(\mathrm{~m}, 3 \mathrm{H}), 8.03(\mathrm{~d}, 2 \mathrm{H}, J=8.2 \mathrm{~Hz}), 8.08(\mathrm{~d}, 1 \mathrm{H}, J=7.2 \mathrm{~Hz}), 12.52($ broad, 1H). ${ }^{13} \mathrm{C} \mathrm{NMR}\left(\mathrm{CD}_{3} \mathrm{SOCD}_{3}\right) \delta_{\mathrm{C}}: 20.4,48.7,63.4,123.0,127.1,127.4,127.7,127.8,129.1,129.2,132.3$, 136.0, 138.5, 144.9, 146.9, 162.6, 170.2. $\mathrm{MS}(\mathrm{m} / \mathrm{z}, \%): 402\left(\mathrm{M}^{+}, 10\right), 383(15), 356(50), 295(40), 252(25)$ 240 (50), 193 (30), 165 (20), 118 (70), 106 (100), 90 (60), 77 (30), 63 (35), 51 (30), 39 (50). Anal. Calcd for $\mathrm{C}_{23} \mathrm{H}_{18} \mathrm{~N}_{2} \mathrm{O}_{5}$ : C, 68.65; H, 4.51; N, 6.96. Found: C, 68.59; H, 4.47; N, 6.87.

cis-3-(4-Bromophenyl)-2-(4-chlorophenyl)-1-oxo-1,2,3,4-tetrahydroisoquinoline-4-carboxlic acid (4i): White powder, yield (406 mg) 89\%, mp 225-6 dec. ${ }^{\circ} \mathrm{C}$, IR $(\mathrm{KBr}),\left(v_{\max } / \mathrm{cm}^{-1}\right): 3065,2920,1723,1630 .{ }^{1} \mathrm{H}$ $\operatorname{NMR}\left(\mathrm{CD}_{3} \mathrm{SOCD}_{3}\right) \delta_{\mathrm{H}}: 4.86\left(\mathrm{~d}, 1 \mathrm{H}, J=4.9 \mathrm{~Hz}, \mathrm{H}_{3}\right), 5.54\left(\mathrm{~d}, 1 \mathrm{H}, J=4.9 \mathrm{~Hz}, \mathrm{H}_{4}\right), 6.94-6.99(\mathrm{~m}, 4 \mathrm{H}), 7.20-7.28$ $(\mathrm{m}, 4 \mathrm{H}), 7.36-7.59(\mathrm{~m}, 5 \mathrm{H}), 8.05(\mathrm{~d}, 1 \mathrm{H}, J=7.5 \mathrm{~Hz}), 2.43(\operatorname{broad}, 1 \mathrm{H}) .{ }^{13} \mathrm{C} \mathrm{NMR}\left(\mathrm{CD}_{3} \mathrm{SOCD}_{3}\right) \delta_{\mathrm{C}}: 48.8$ $63.1,127.5,127.6,127.8,128.4,128.5,128.9,129.2,129.9,130.8,131.0,132.4,134.1,136.4,139.3,162.8$ 170.2. MS (m/z,\%): $456\left(\mathrm{M}^{+}, 15\right), 412(55), 345$ (50), $301(60), 267$ (45), $257(35), 223(25), 193(30), 178$ (20), 118 (60), 106 (100), 90 (50), 77 (35), 63 (30), 51 (30), 39 (55). Anal. Calcd for $\mathrm{C}_{22} \mathrm{H}_{15} \mathrm{NO}_{3} \mathrm{BrCl}: \mathrm{C}_{\text {, }}$ 57.86; H, 3.31; N, 3.07. Found: C, 57.78; H, 3.22; N, 2.98.

cis-3-(3-Chlorophenyl)-2-(4-chlorophenyl)-1-oxo-1,2,3,4-tetrahydroisoquinoline-4-carboxlic acid (4I): White powder, yield $(357 \mathrm{mg}) 87 \%, \mathrm{mp} 210-1 \mathrm{dec} .{ }^{\circ} \mathrm{C}, \mathrm{IR}(\mathrm{KBr}),\left(\mathrm{v}_{\max } / \mathrm{cm}^{-1}\right): 3040,2915,1722,1630 .{ }^{1} \mathrm{H}$ $\operatorname{NMR}\left(\mathrm{CD}_{3} \mathrm{SOCD}_{3}\right) \delta_{\mathrm{H}}: 4.83\left(\mathrm{~d}, 1 \mathrm{H}, J=5.3 \mathrm{~Hz}, \mathrm{H}_{3}\right), 5.60\left(\mathrm{~d}, 1 \mathrm{H}, J=5.3 \mathrm{~Hz}, \mathrm{H}_{4}\right), 7.01(\mathrm{~d}, 1 \mathrm{H}, J=7.2 \mathrm{~Hz}), 7.10$ (s, 1H), 7.20-7.26 (m, 4H), 7.36-7.38 (m, 2H), 7.49-7.62 (m, 3H), 8.05 (d, 1H, J=7.7), 12.53 (broad, 1H). ${ }^{13} \mathrm{C}$ NMR $\left(\mathrm{CD}_{3} \mathrm{SOCD}_{3}\right) \delta_{\mathrm{C}}: 49.0,62.9,126.5,127.5,127.7,127.8,127.9,128.0,128.4,128.5,129.1,129.9$ 130.7, 132.5, 134.2, 139.5, 139.9, 162.9, 170.3. MS (m/z, \%): $411\left(\mathrm{M}^{+}, 15\right), 367(25), 333(40), 299(45)$ 257 (45), 244 (50), 223 (35), 178 (35), 165 (25), 118 (100), $111(25), 90(45), 77$ (30), 63 (30), 51 (35), 39 (50). Anal. Calcd for $\mathrm{C}_{22} \mathrm{H}_{15} \mathrm{NO}_{3} \mathrm{Cl}_{2}$ : C, 64.09; H, 3.67; N, 3.40. Found: C, 64.00; H, 3.57; N, 3.32. 
cis-2-Benzyl-3-(2-bromophenyl)-1-oxo-1,2,3,4-tetrahydroisoquinoline-4-carboxlic acid (4m): White powder, yield (352 mg) 81\%, mp 216-8 ${ }^{\circ} \mathrm{C}$, IR $(\mathrm{KBr}),\left(v_{\max } / \mathrm{cm}^{-1}\right): 3060,2925,1735,1610 .{ }^{1} \mathrm{H} \mathrm{NMR}$ $\left(\mathrm{CD}_{3} \mathrm{SOCD}_{3}\right) \delta_{\mathrm{H}}: 3.71\left(\mathrm{~d}, 1 \mathrm{H}, J=15.2, \mathrm{CH}_{2}\right), 4.61\left(\mathrm{~d}, 1 \mathrm{H}, J=6.5 \mathrm{~Hz}, \mathrm{H}_{3}\right), 5.32\left(\mathrm{~d}, 1 \mathrm{H}, J=15.2 \mathrm{~Hz}, \mathrm{CH}_{2}\right), 5.43$ (d, 1H, J=6.5 Hz, H4), $6.95(\mathrm{~m}, 1 \mathrm{H}), 7.14-7.34(\mathrm{~m}, 8 \mathrm{H}), 7.50-7.60(\mathrm{~m}, 3 \mathrm{H}), 8.08(\mathrm{~d}, 1 \mathrm{H}, J=7.6 \mathrm{~Hz}), 12.50$ (broad, $1 \mathrm{H}) .{ }^{13} \mathrm{C} \mathrm{NMR}\left(\mathrm{CD}_{3} \mathrm{SOCD}_{3}\right) \delta_{\mathrm{C}}: 47.6,47.7,58.0,124.3,127.1,127.3,127.4,127.5,127.7,128.0$, 128.2, 128.3, 128.6, 130.0, 132.3, 132.5, 133.6, 135.9, 136.7, 162.9, 169.9. MS (m/z, \%): $435\left(\mathrm{M}^{+}, 15\right), 391$ (15), 356 (50), 345 (10), 312 (35), 302 (10), 278 (35), 261 (10), 234 (40), 206 (45), 178 (55), 118 (20), 106 (35), 91 (100), 65 (45), 51 (35), 39 (30). Anal. Calcd for $\mathrm{C}_{23} \mathrm{H}_{18} \mathrm{NO}_{3} \mathrm{Br}$ : C, 63.32; H, 4.16; N, 3.21. Found: C, 63.23; H, 4.10; N, 3.13. 


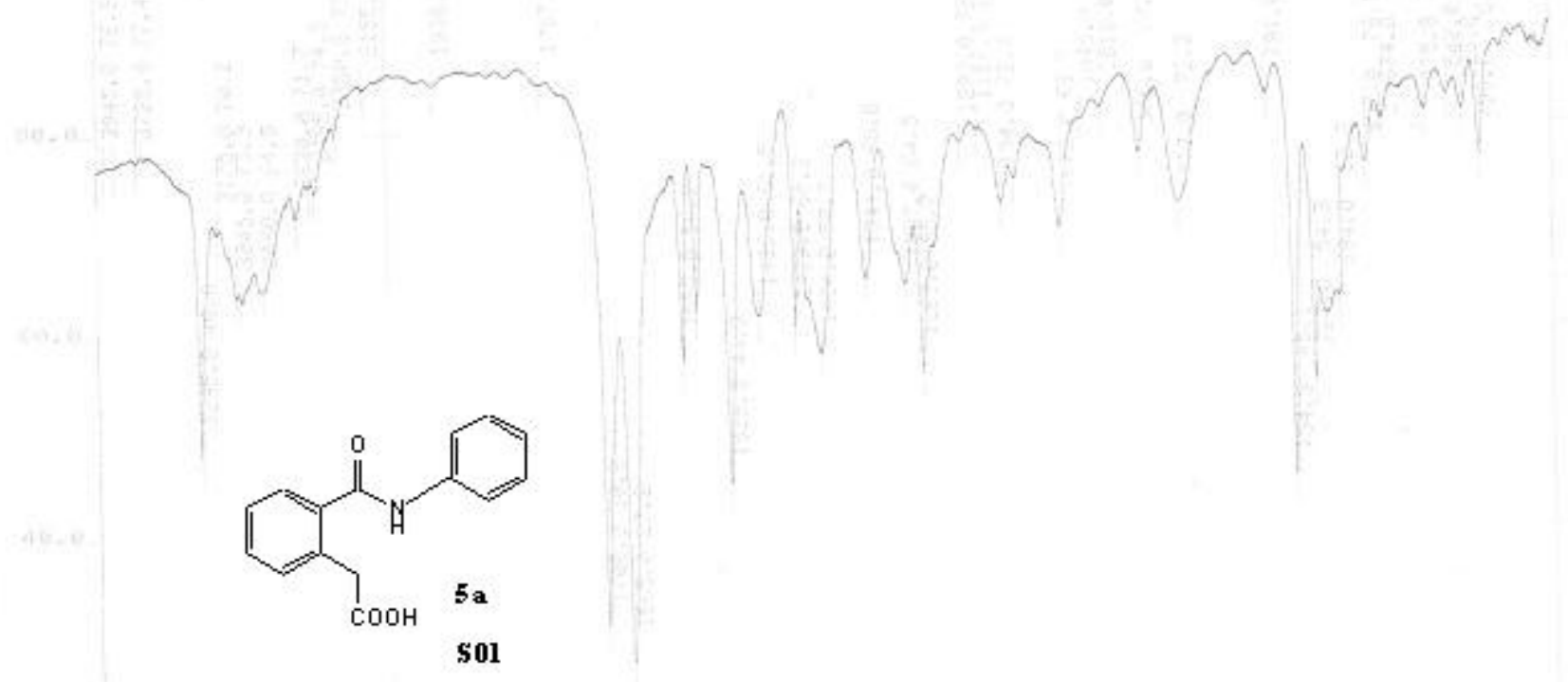


HA17

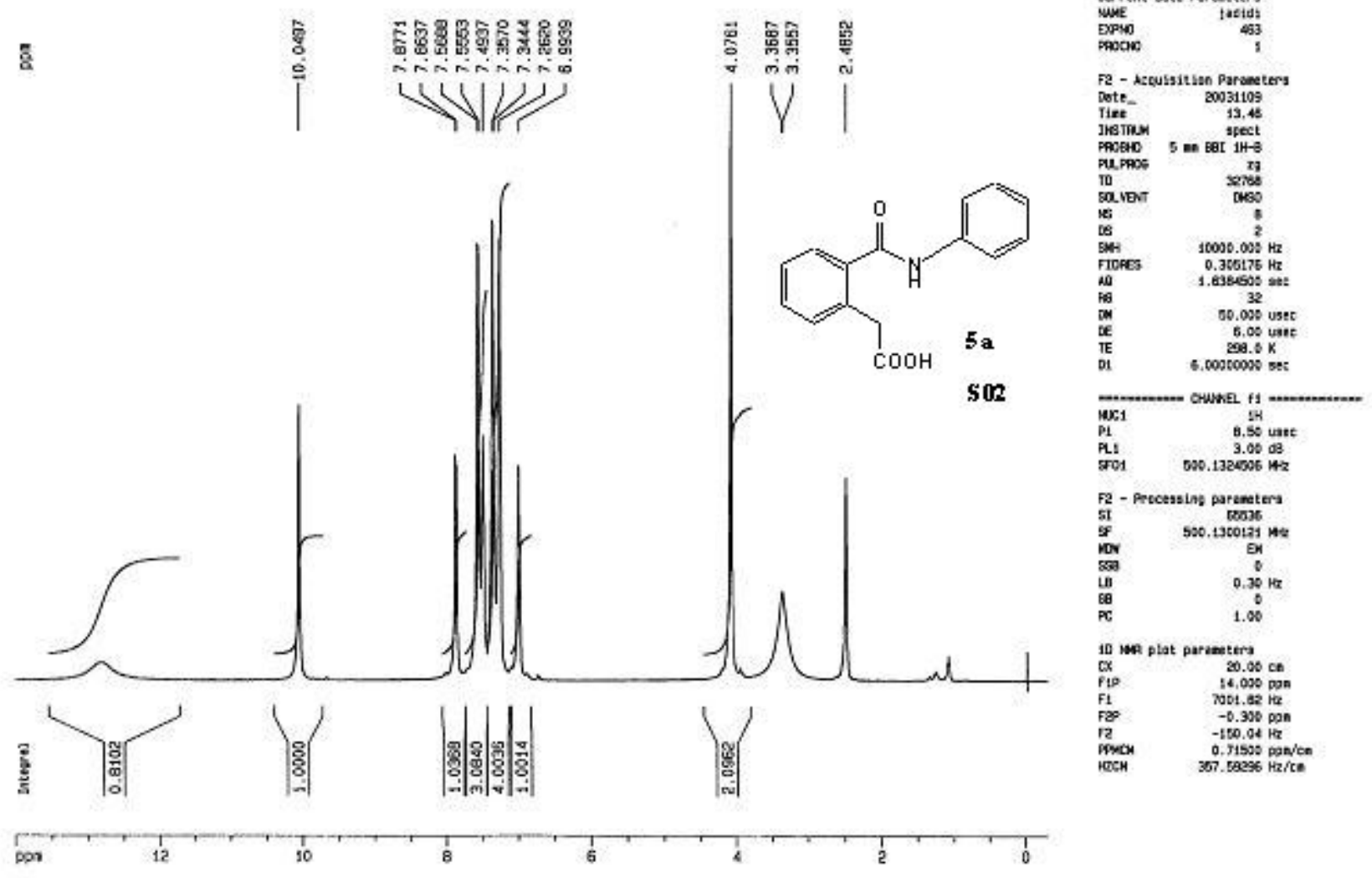


700.9

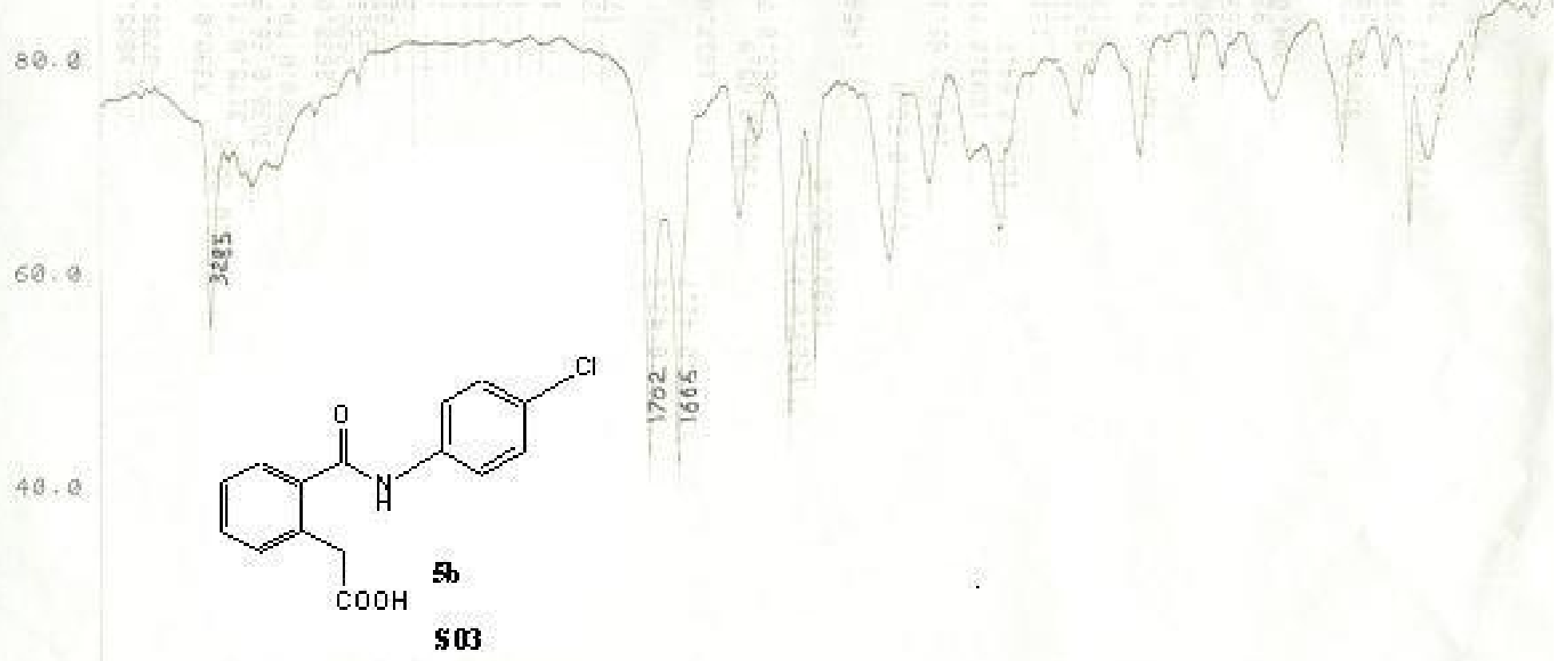

20.0

0.0

4090.8

3ege.

2808,8

1500.0

tese. 0 


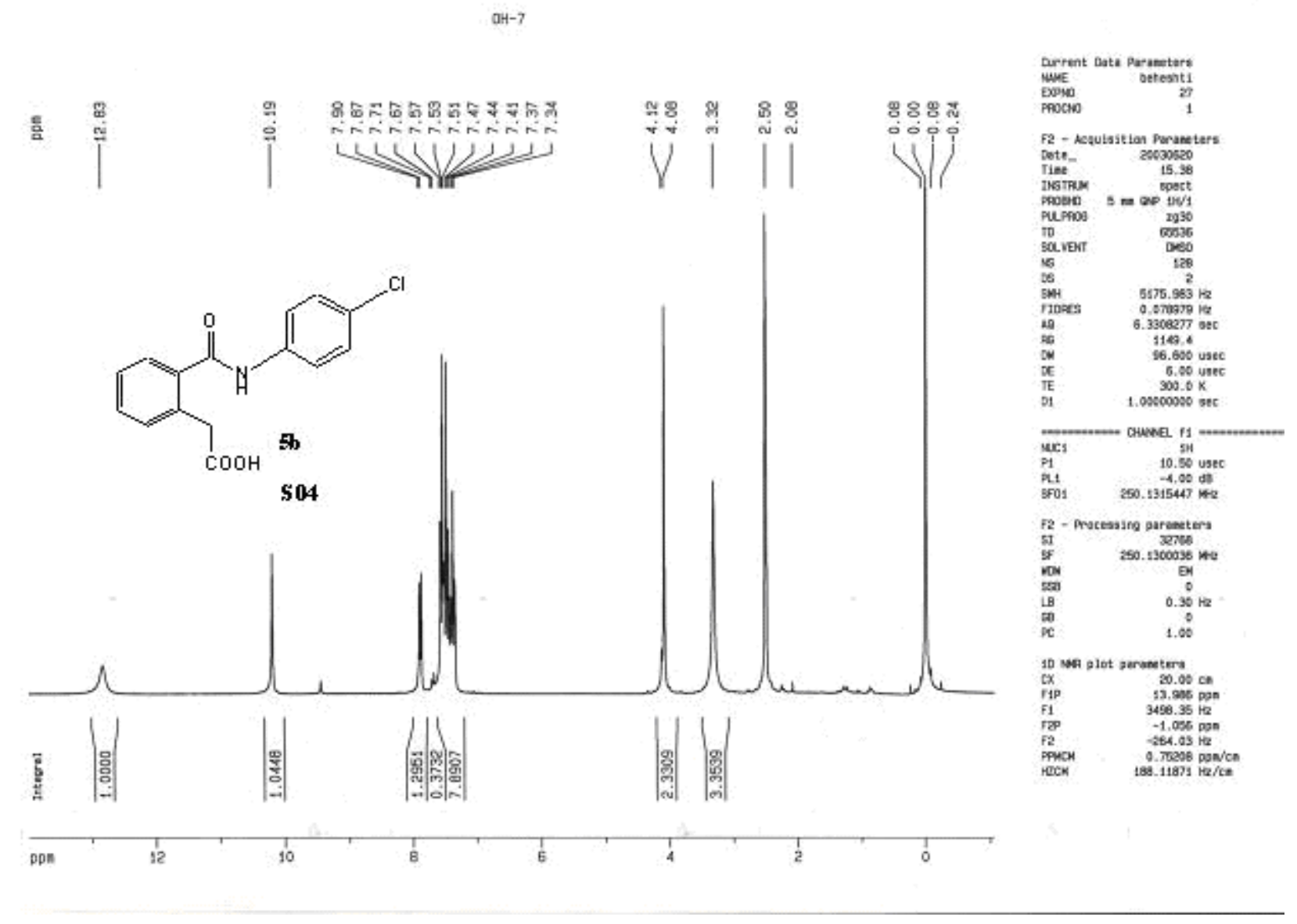



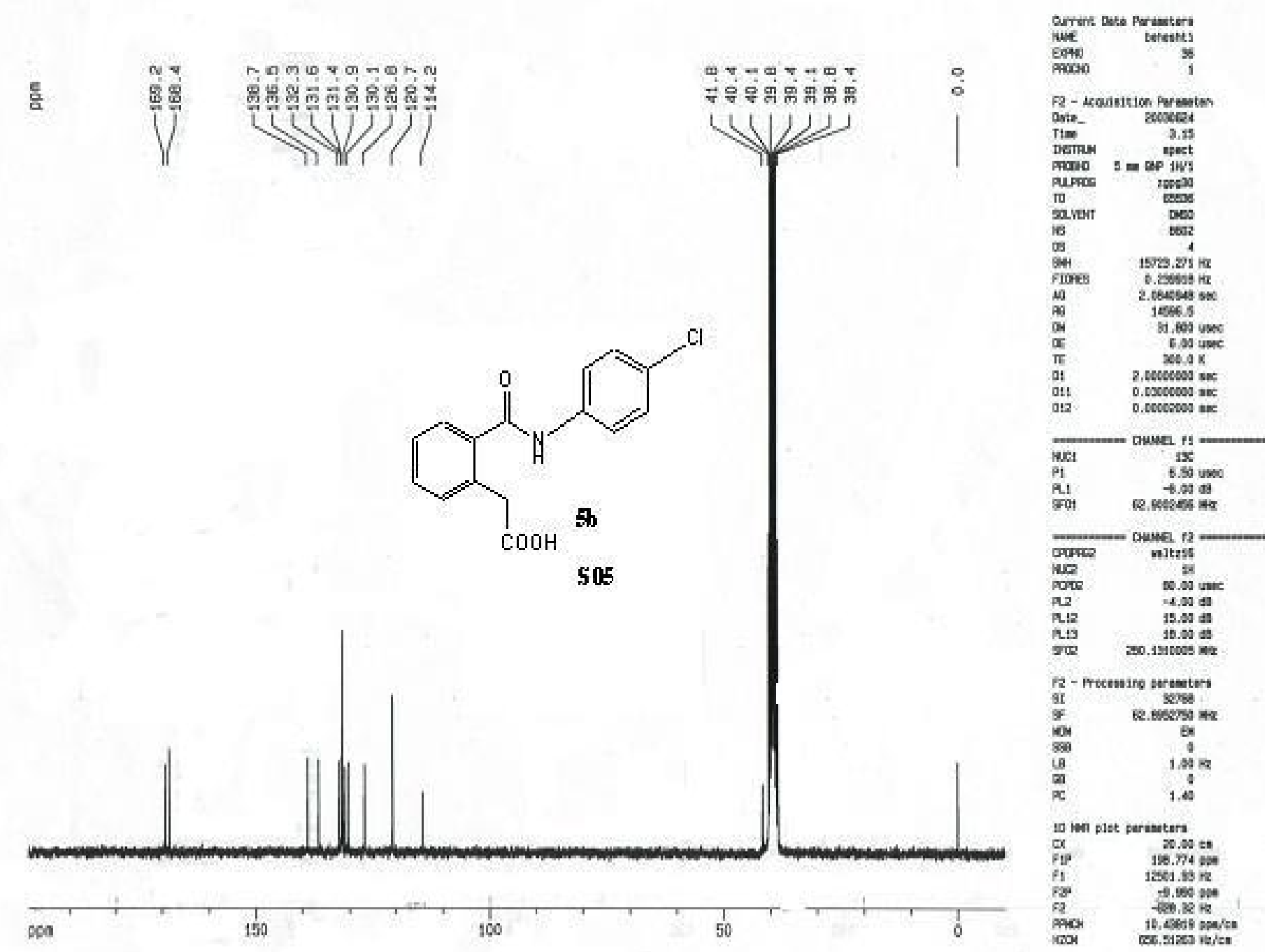
100,0

100.6

B8. 8

$2 \theta \cdot \theta$

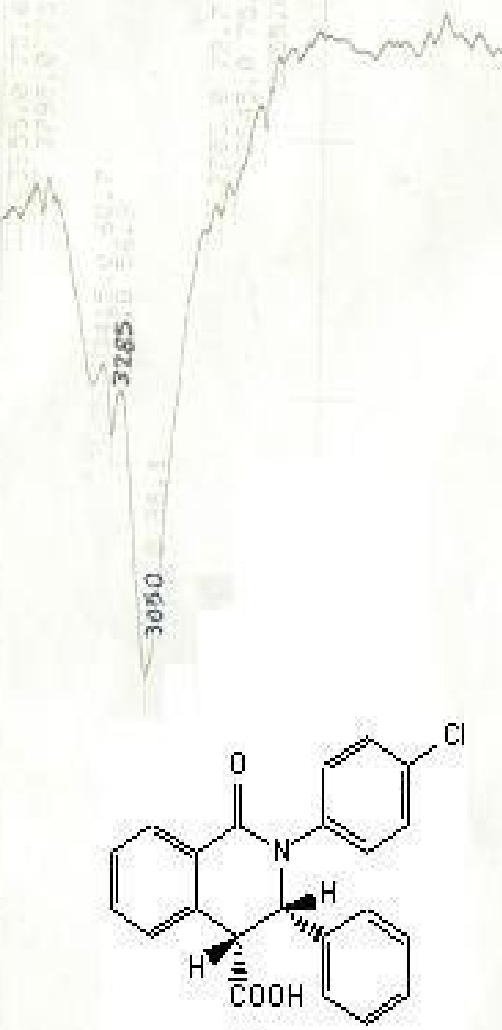

4

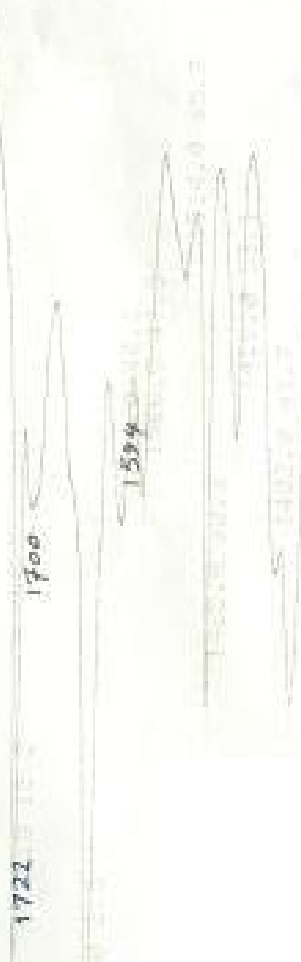

2
0

$\mathbf{5 0 6}$

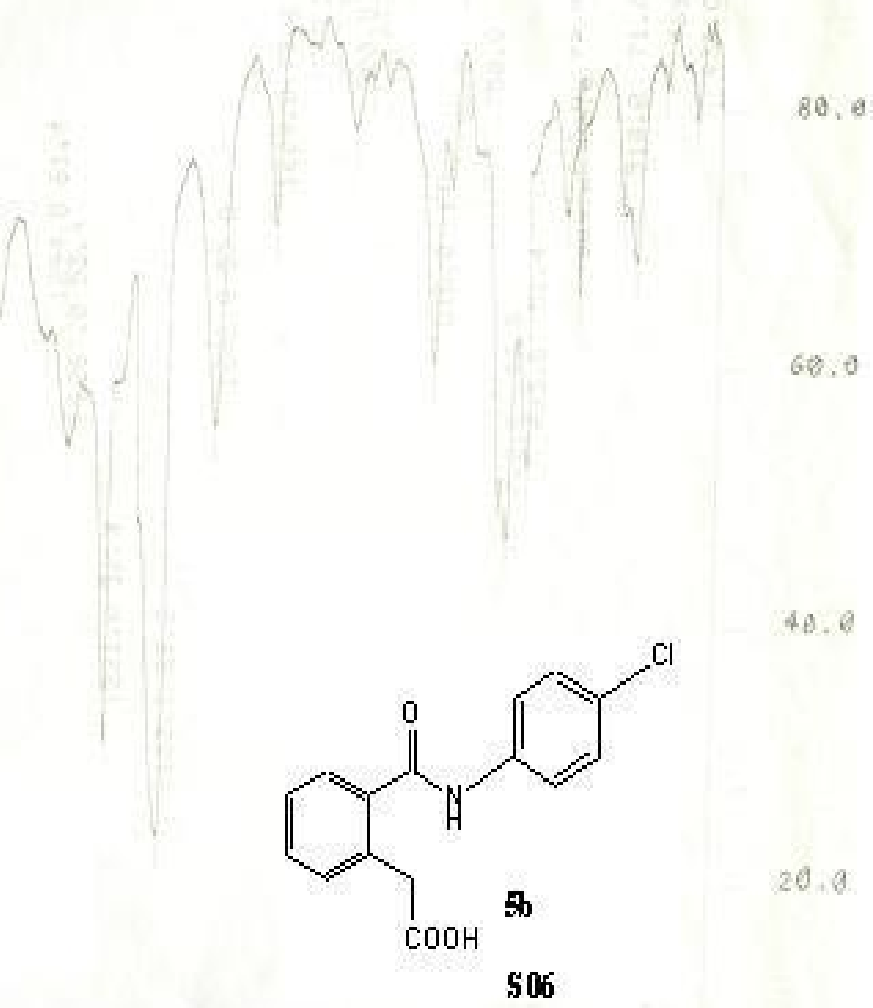

$-8.0$

$2000+\theta$

1500.8

1800.6

$\theta \cdot \theta$

580.8 408. 


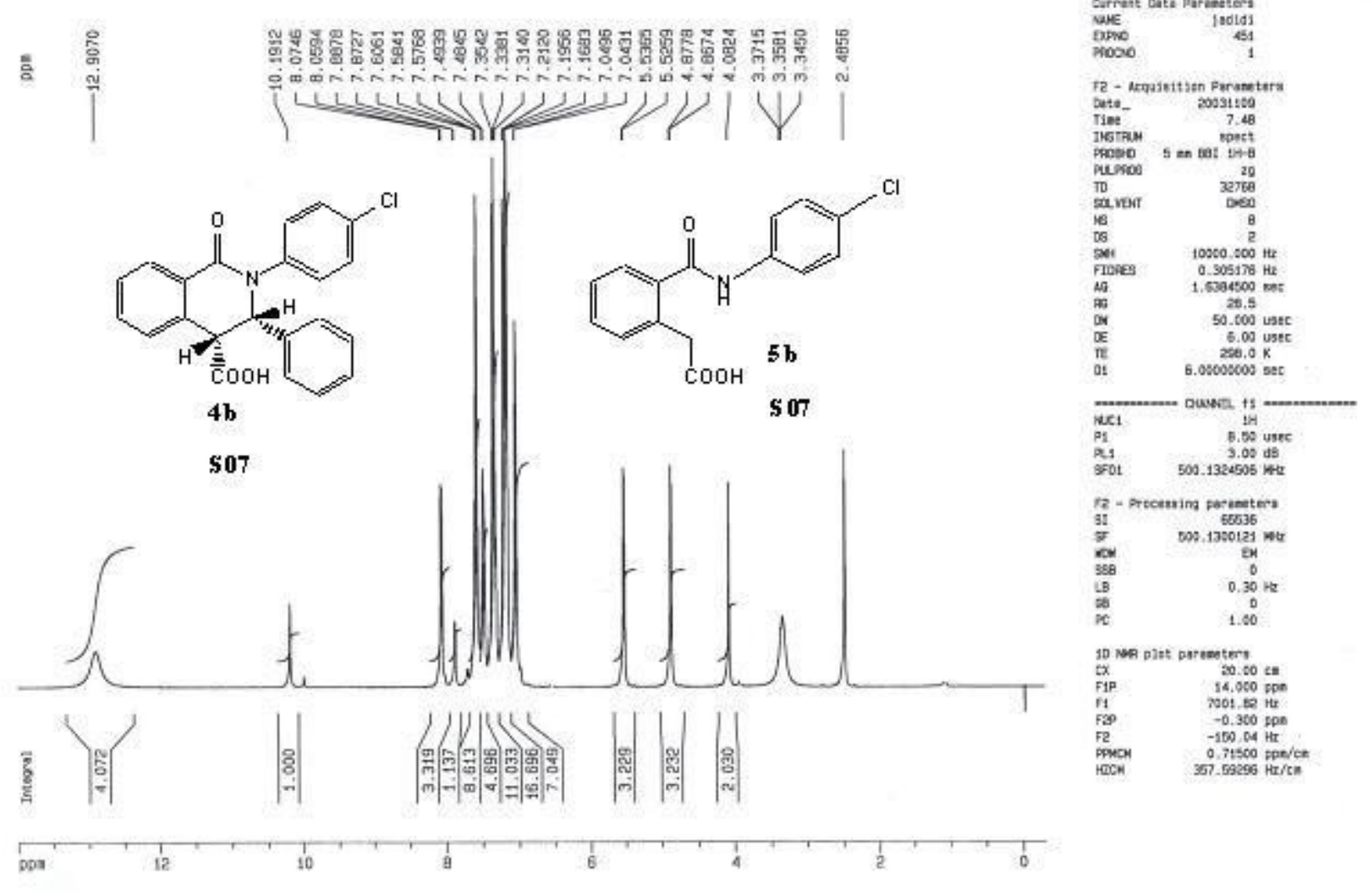



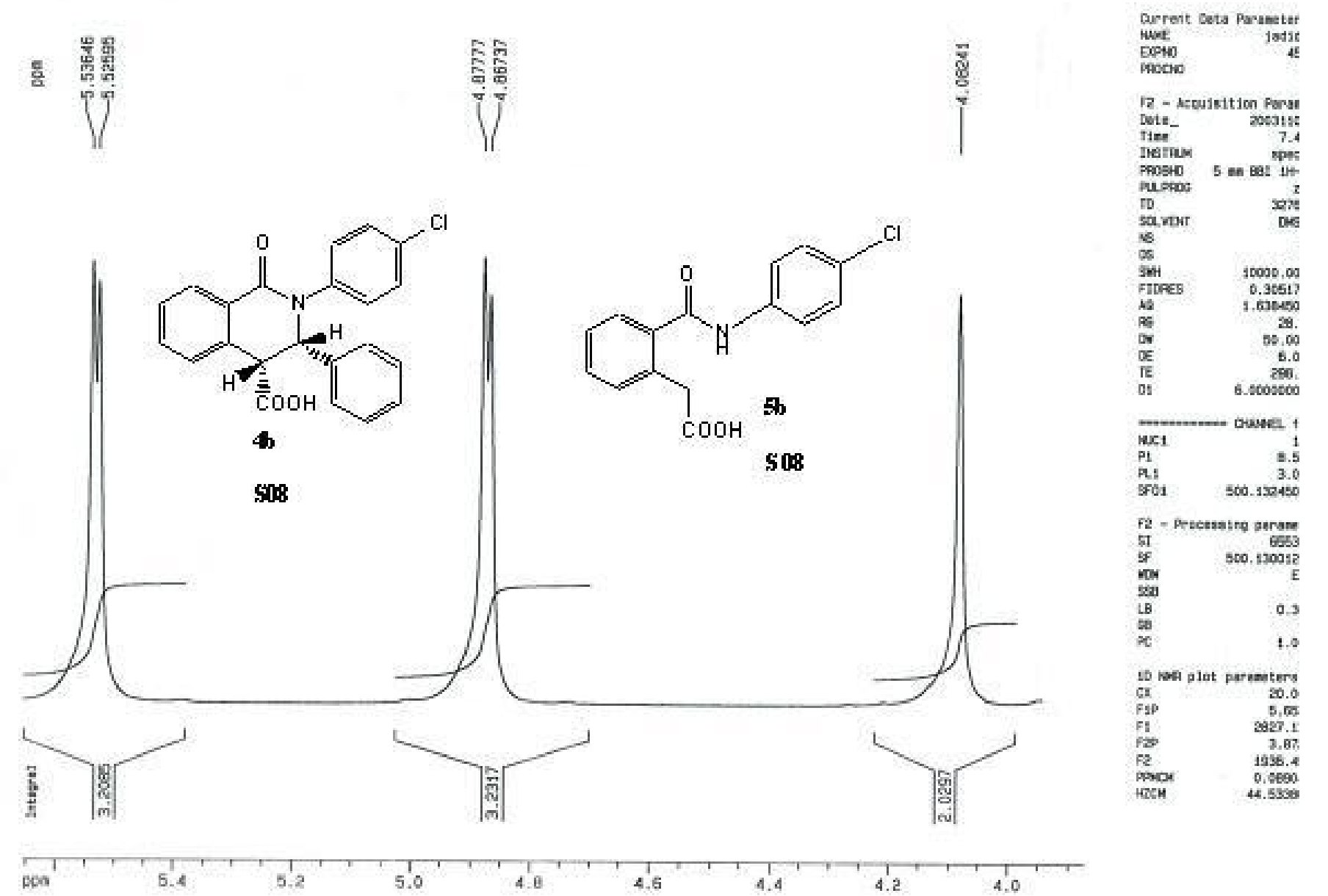

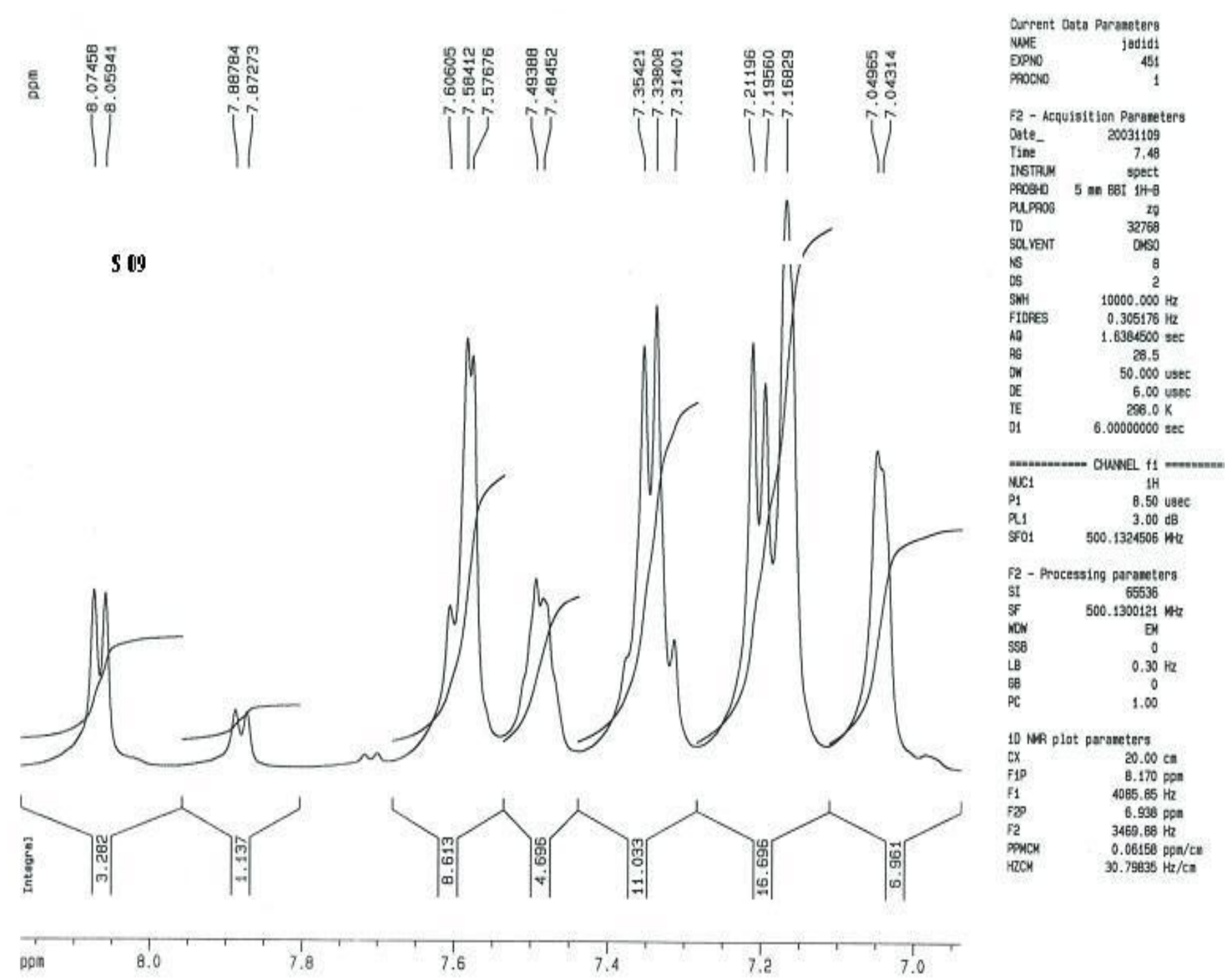


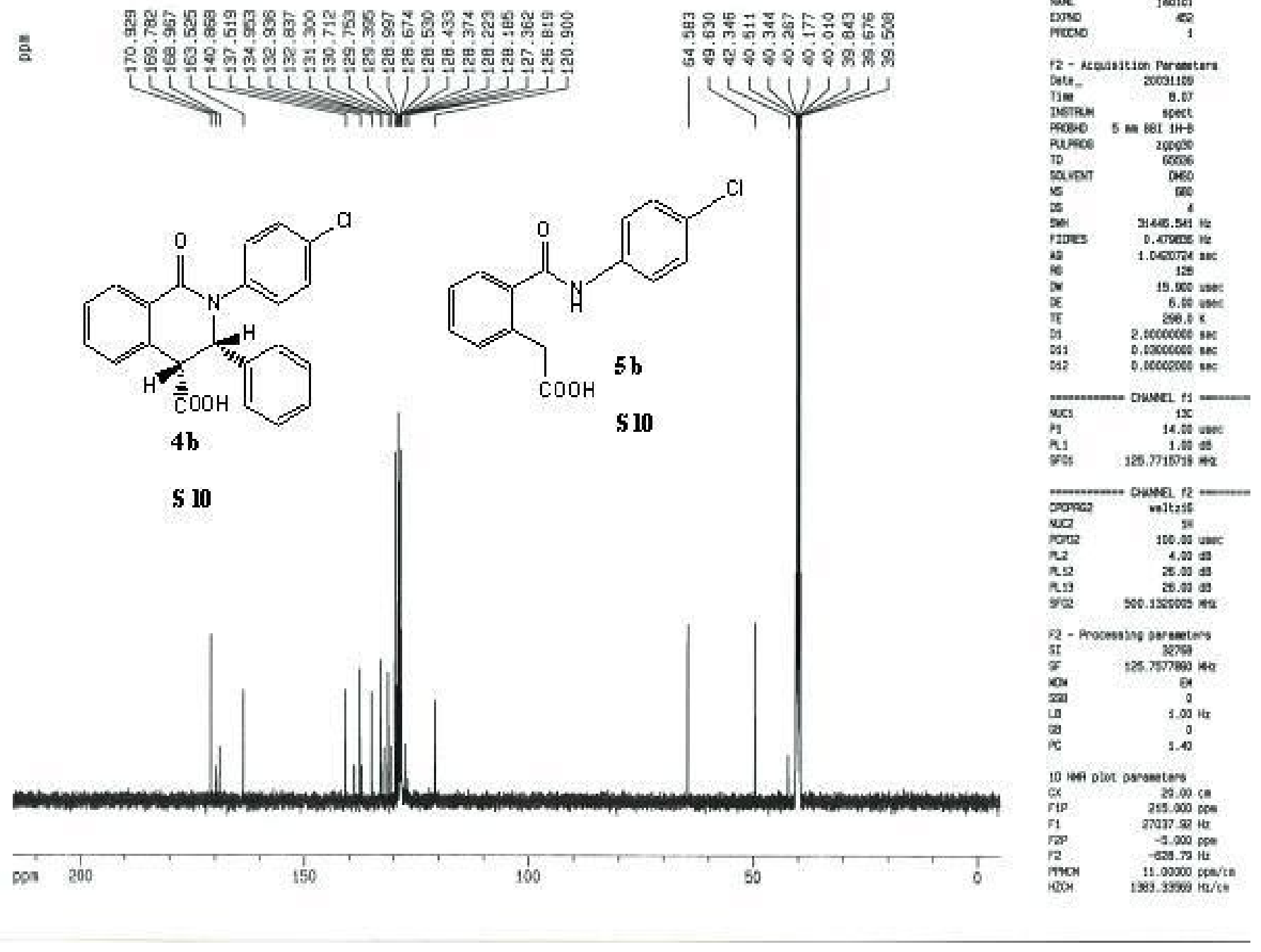



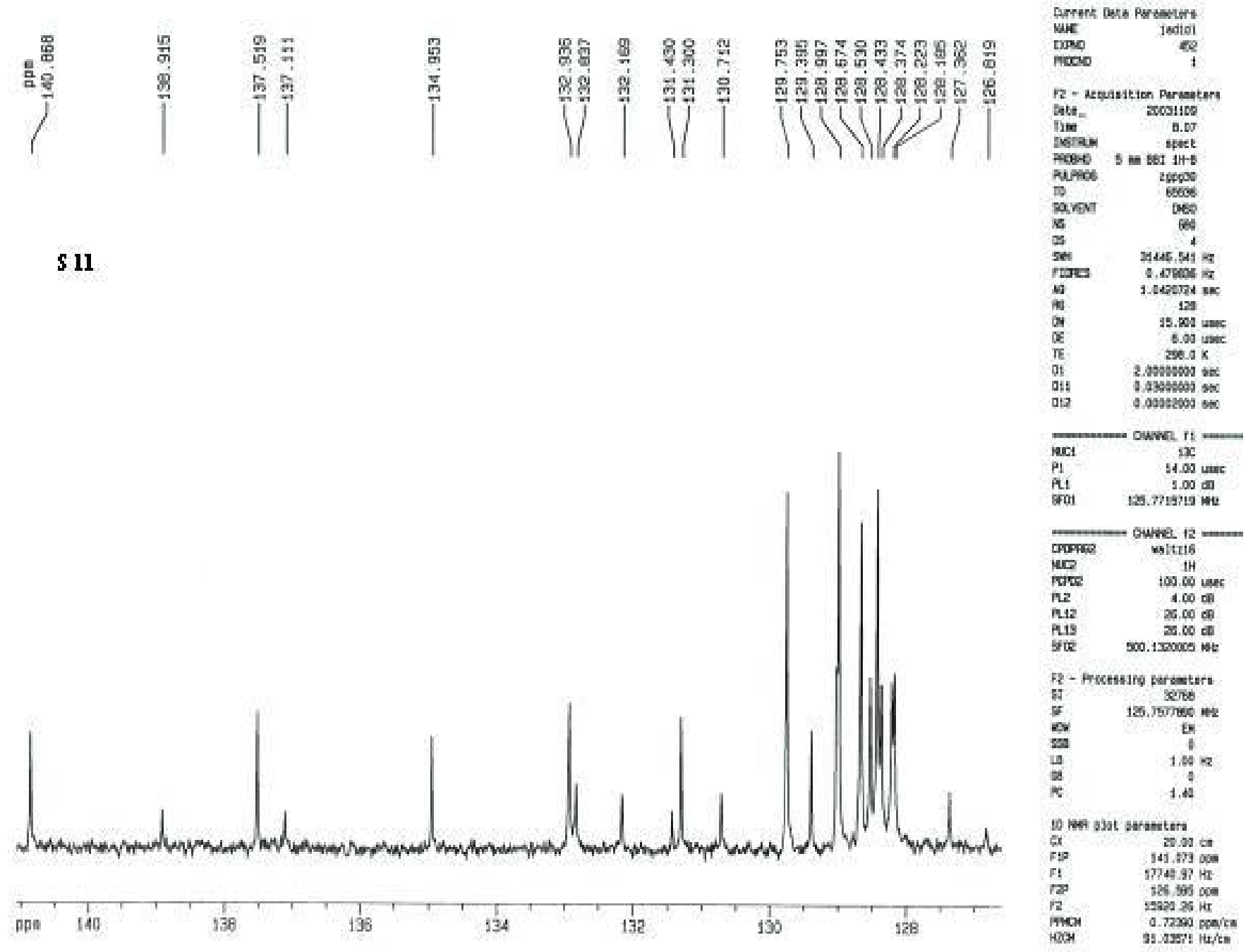


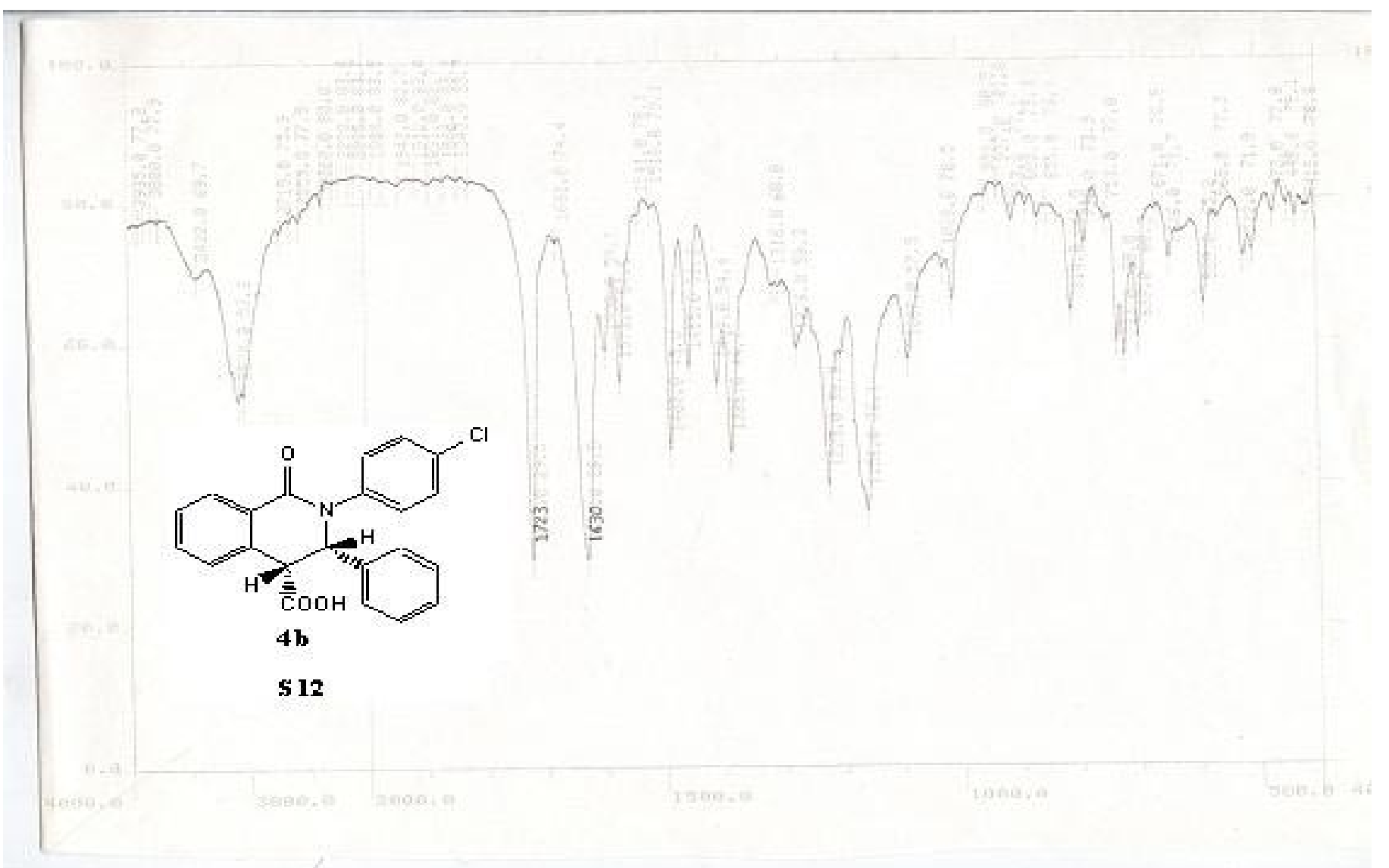



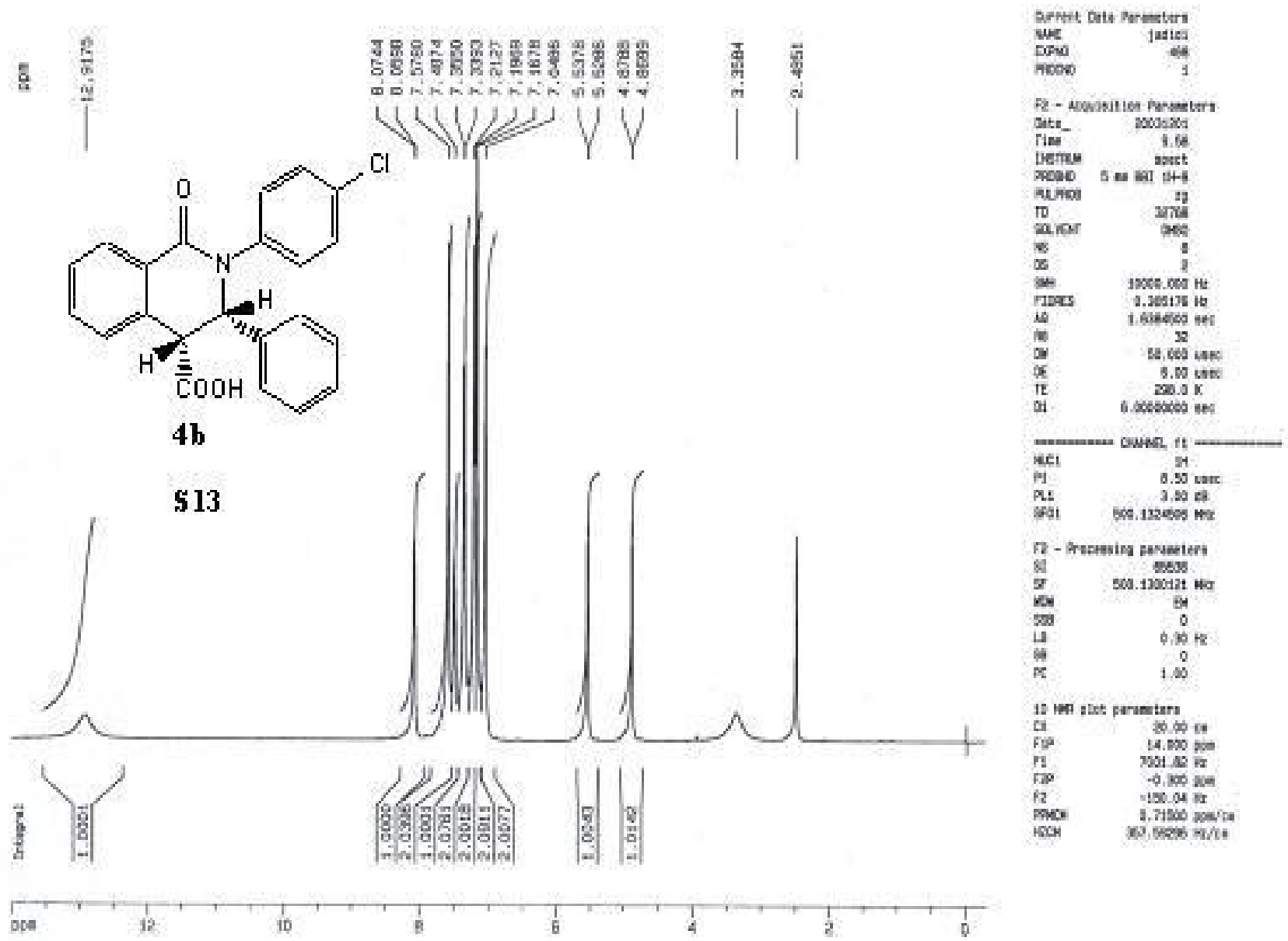


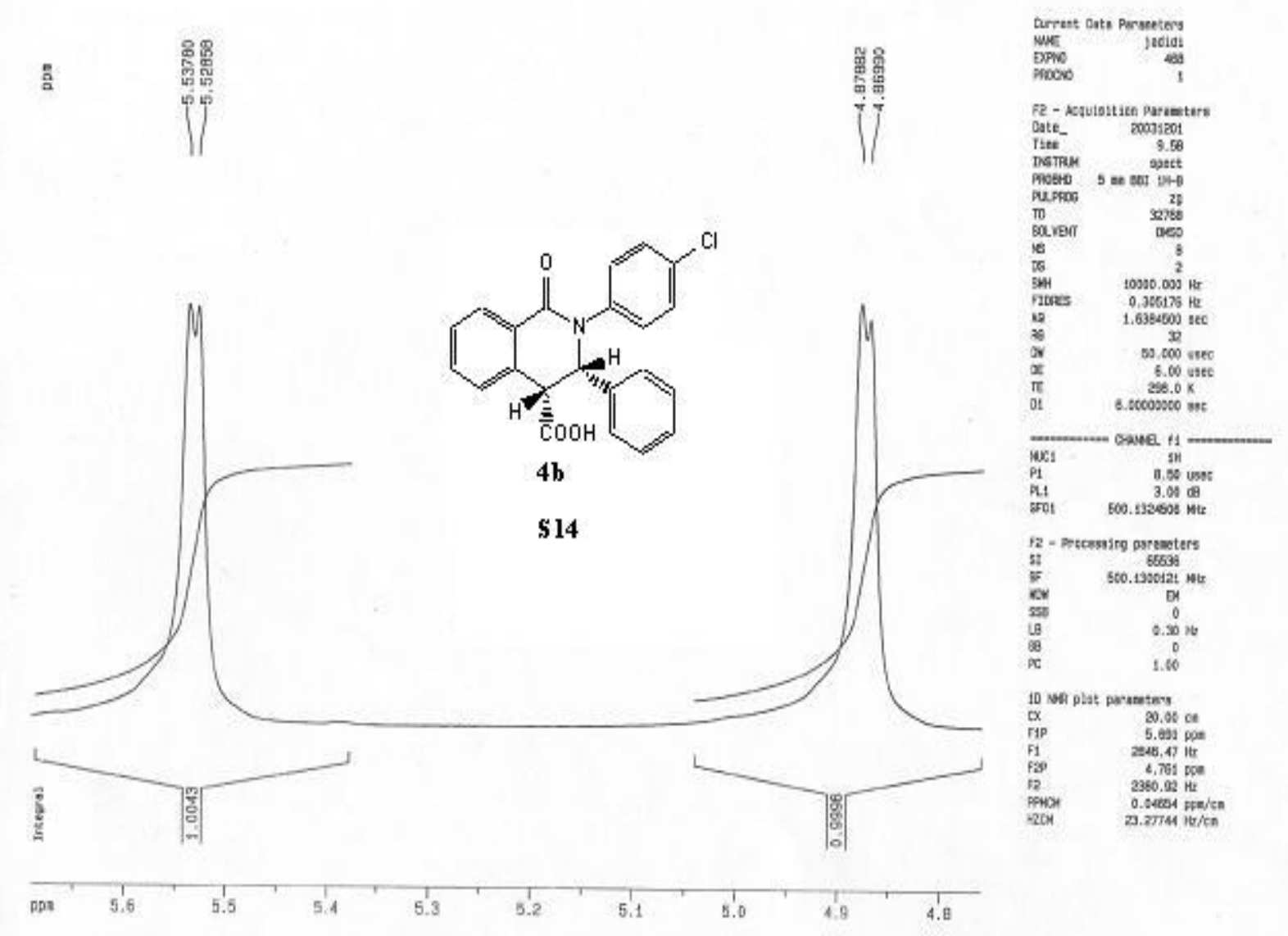




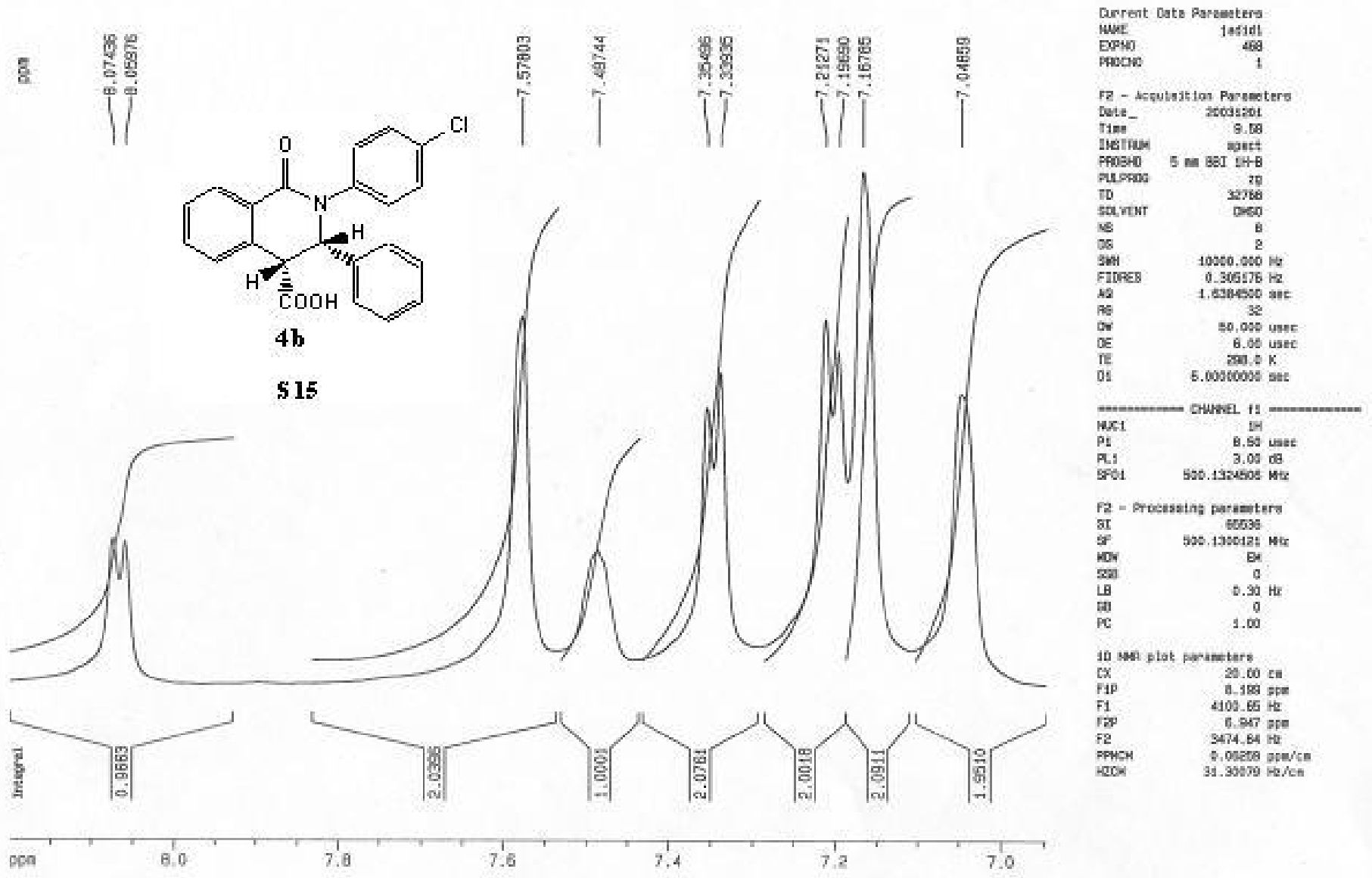




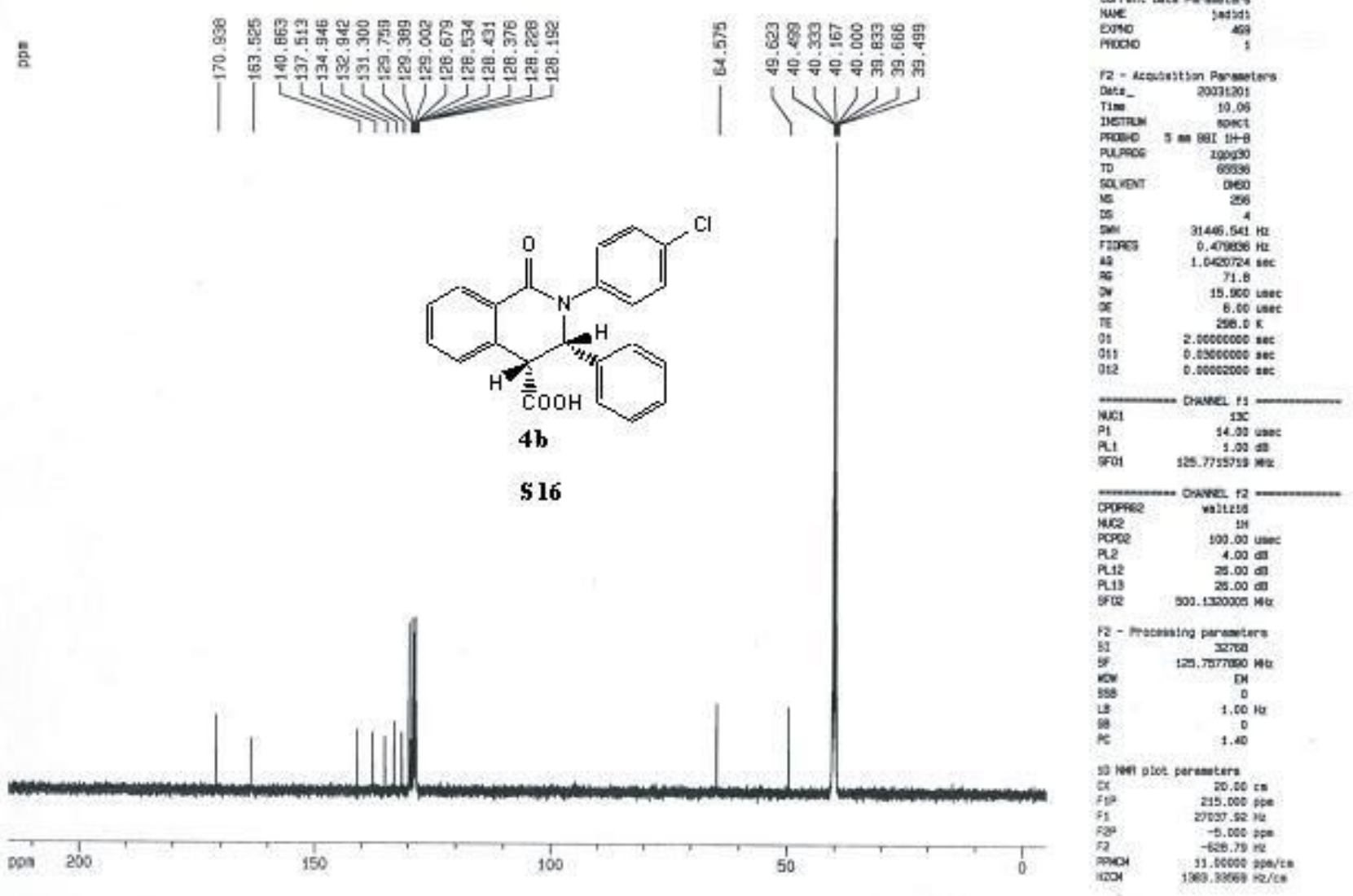




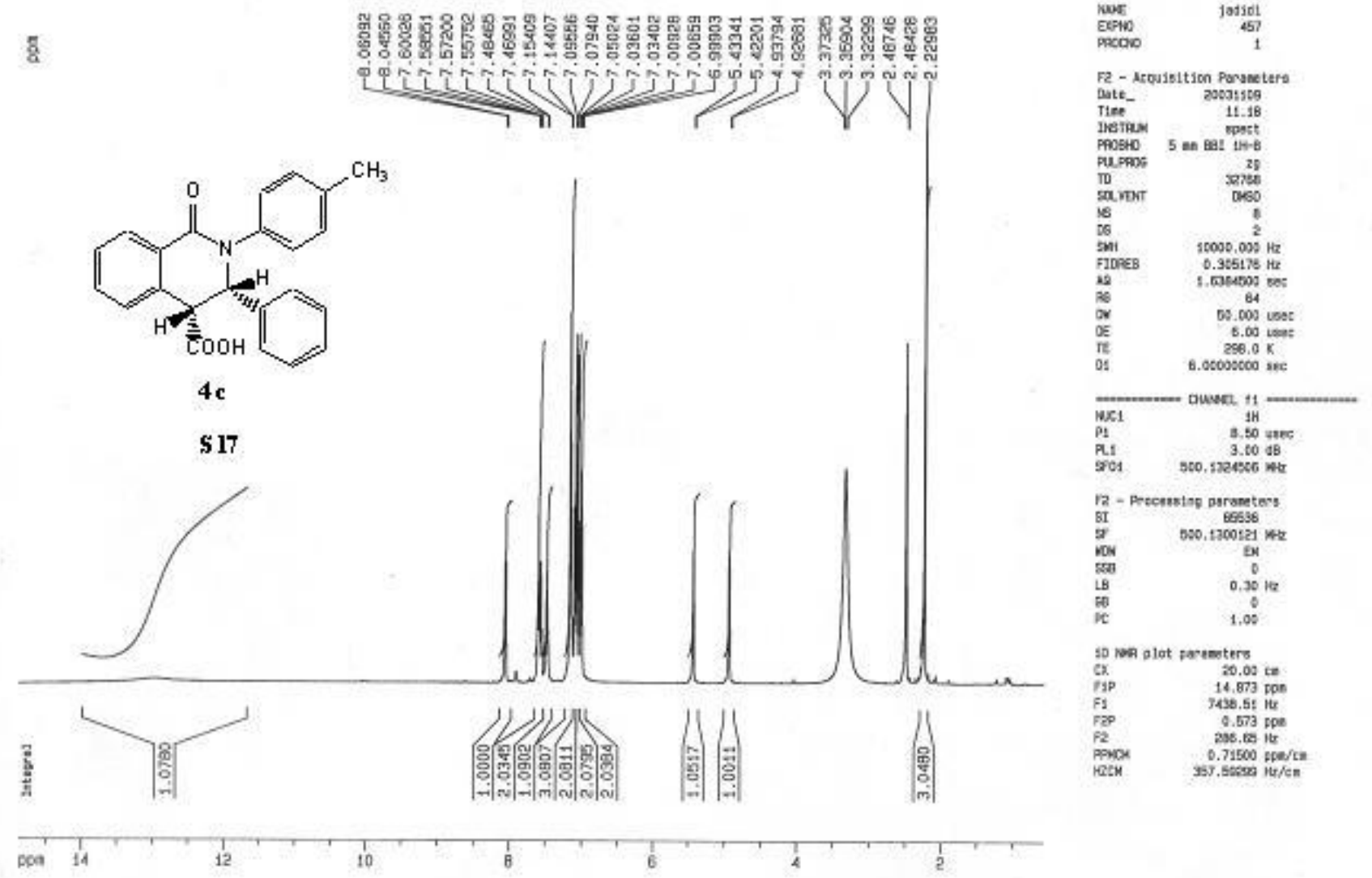




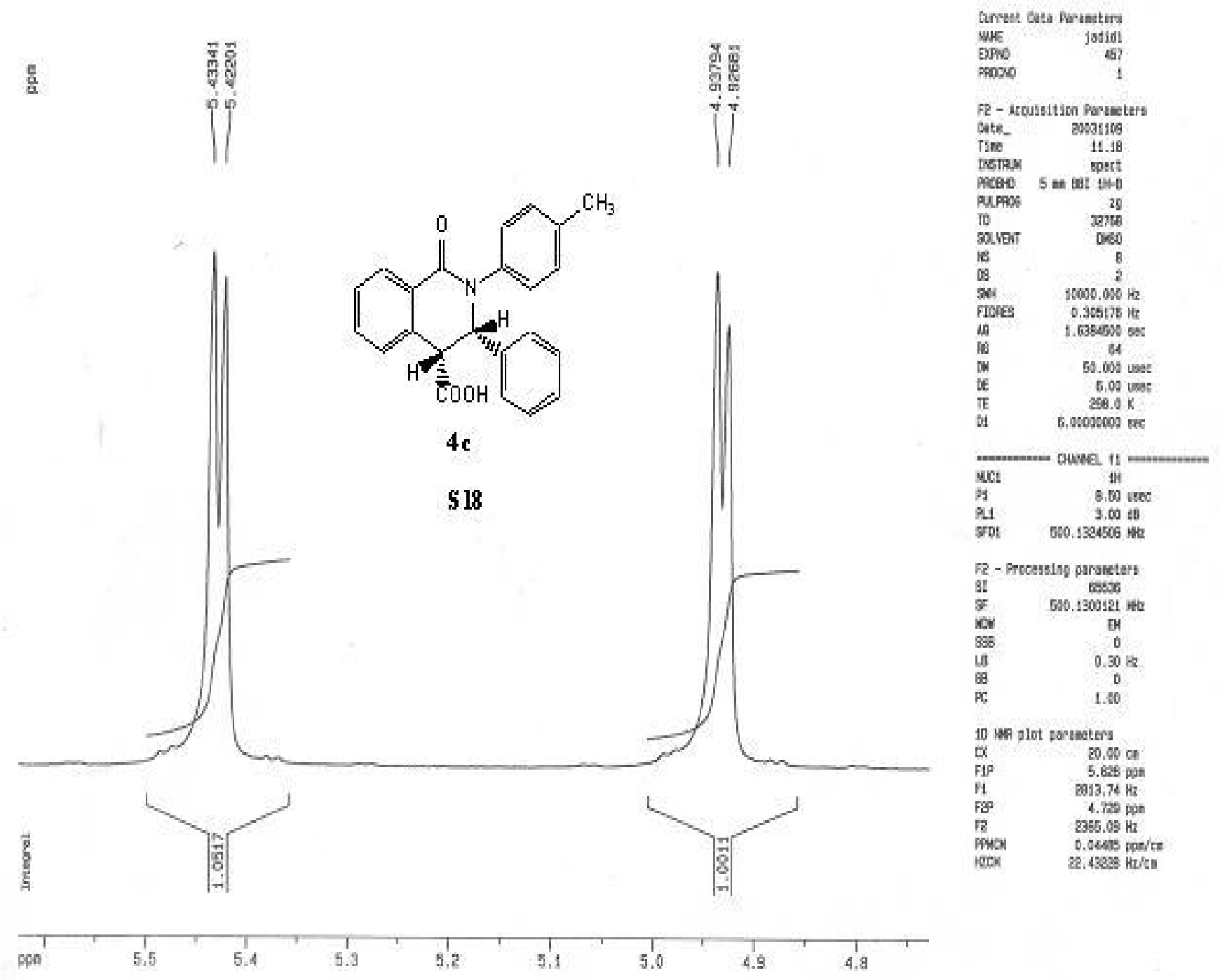



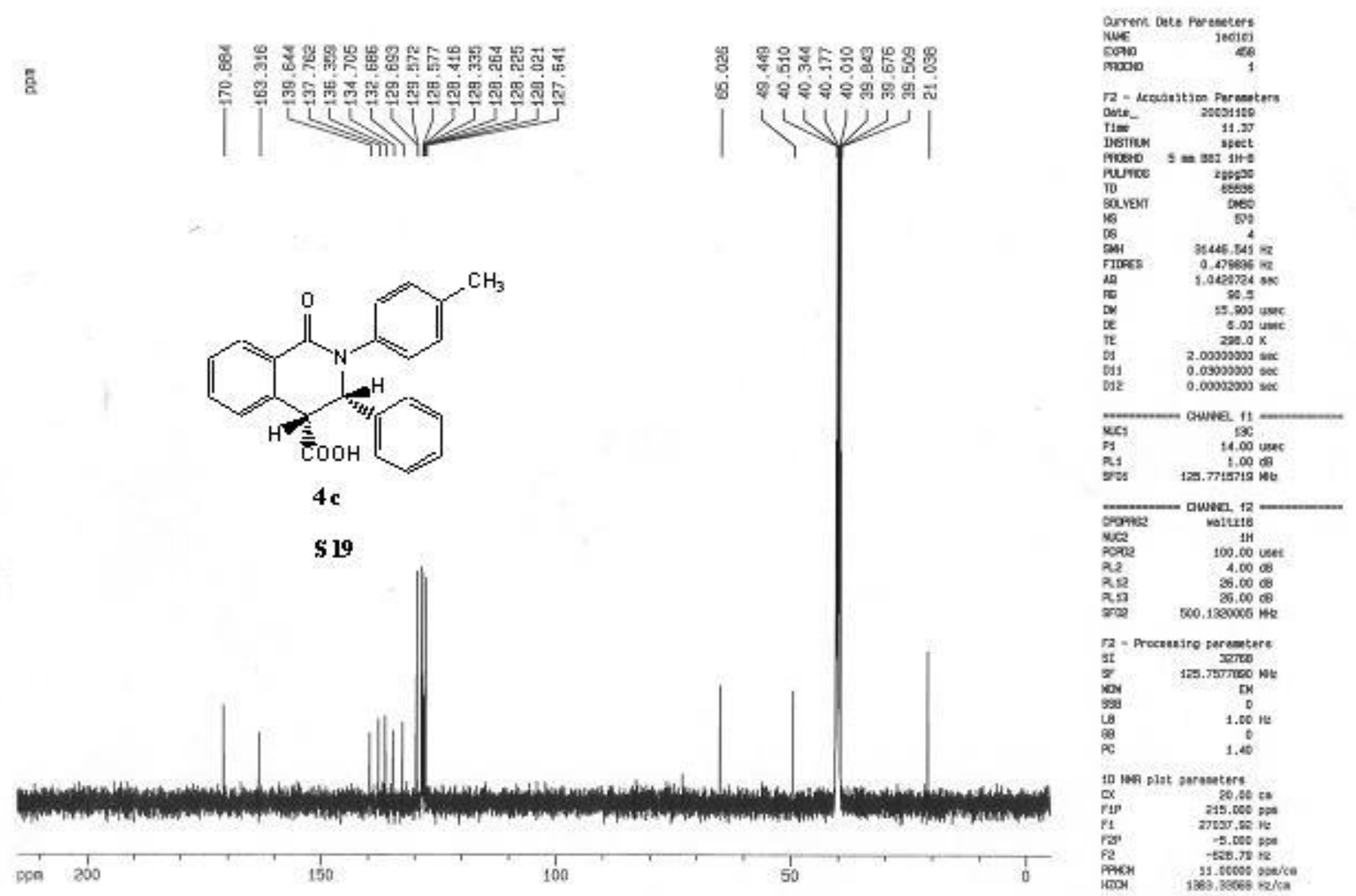


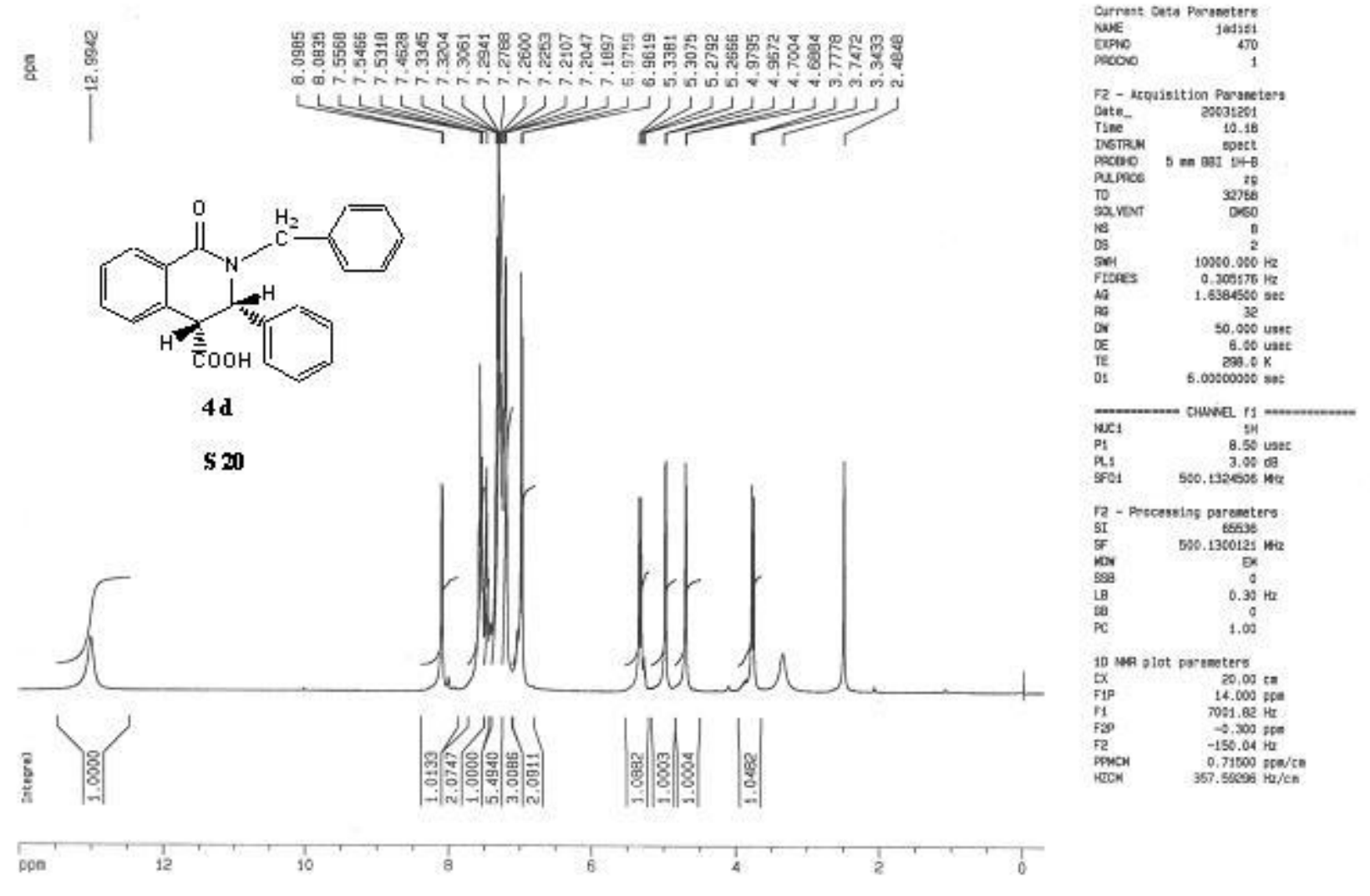



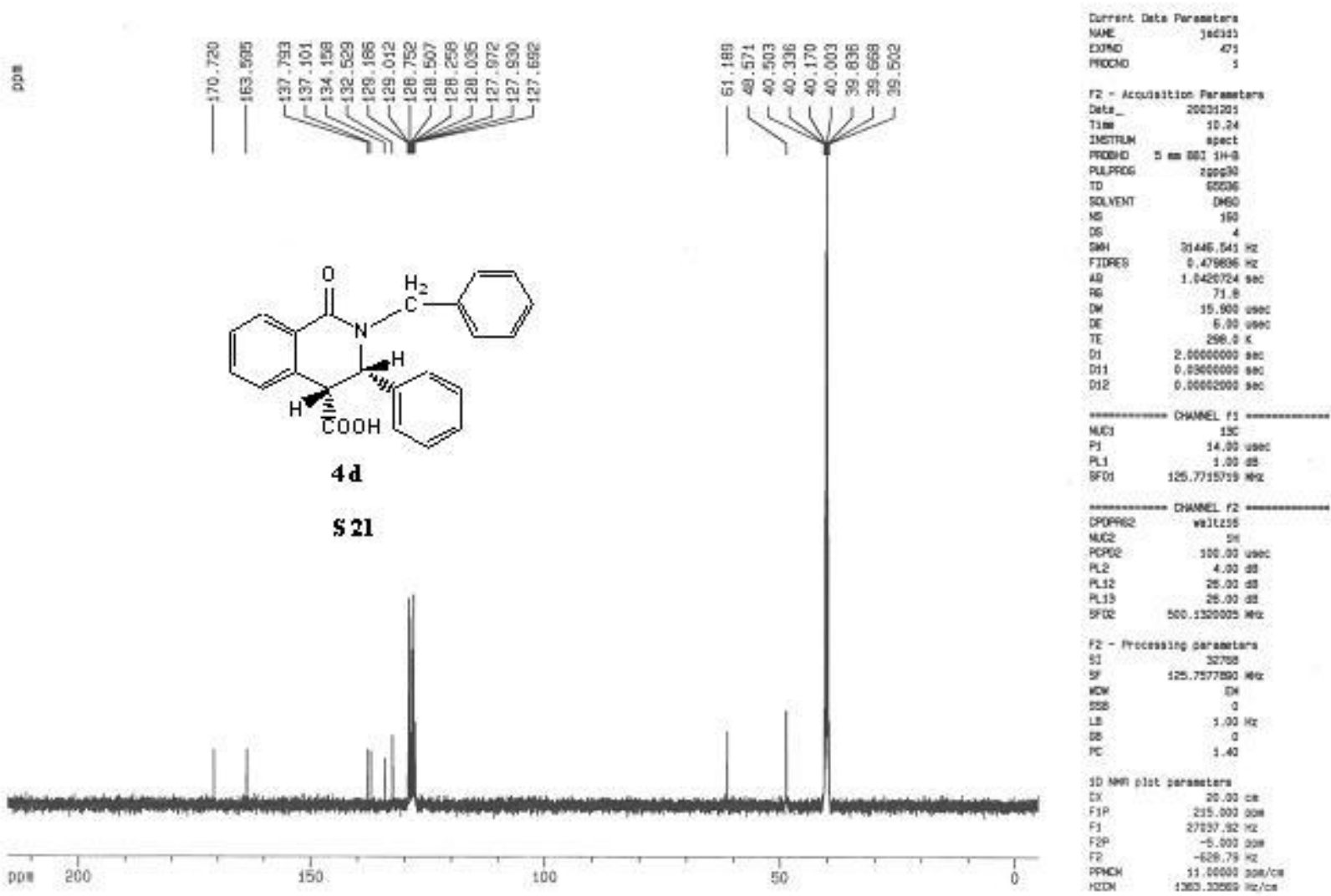


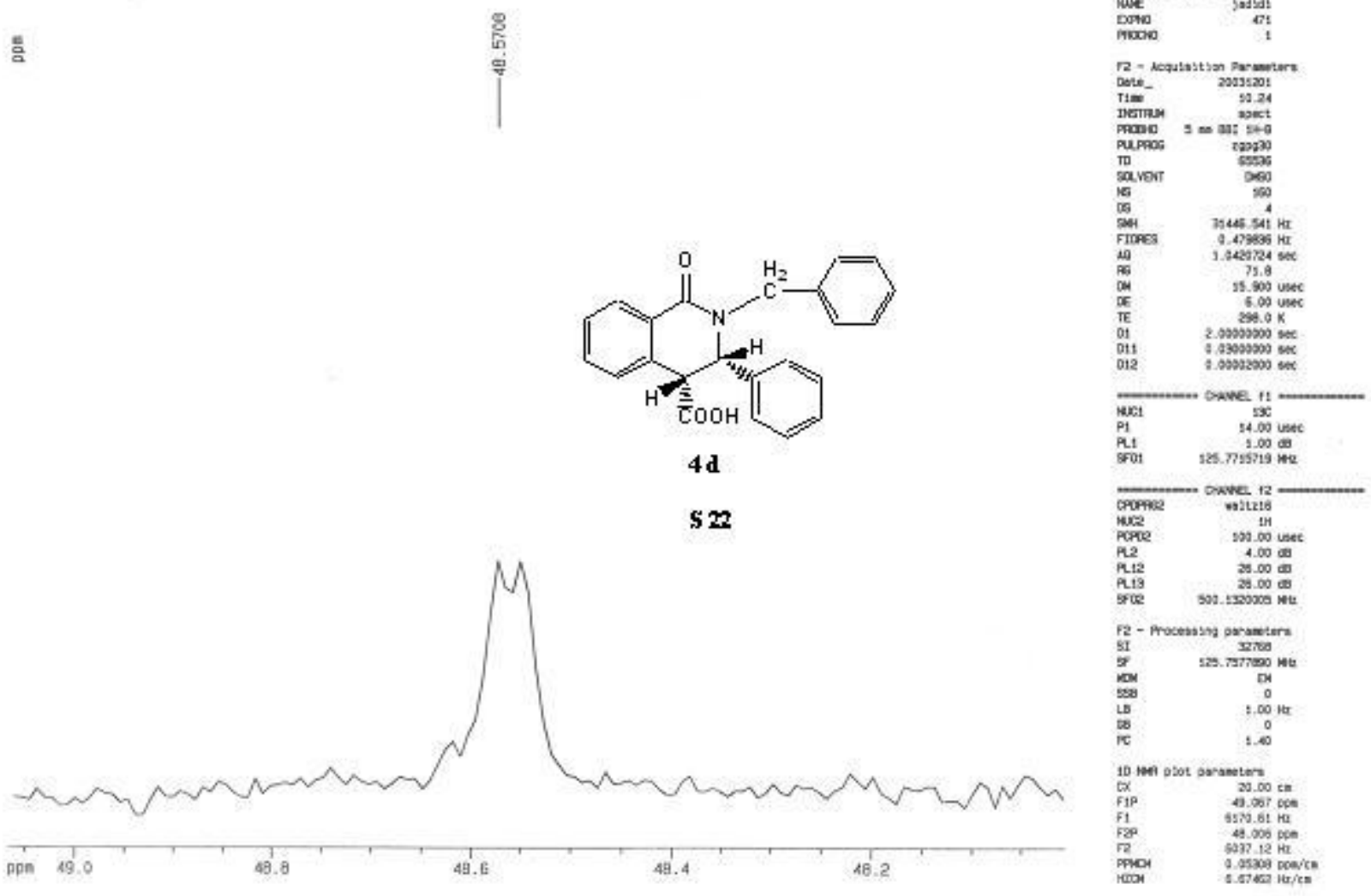



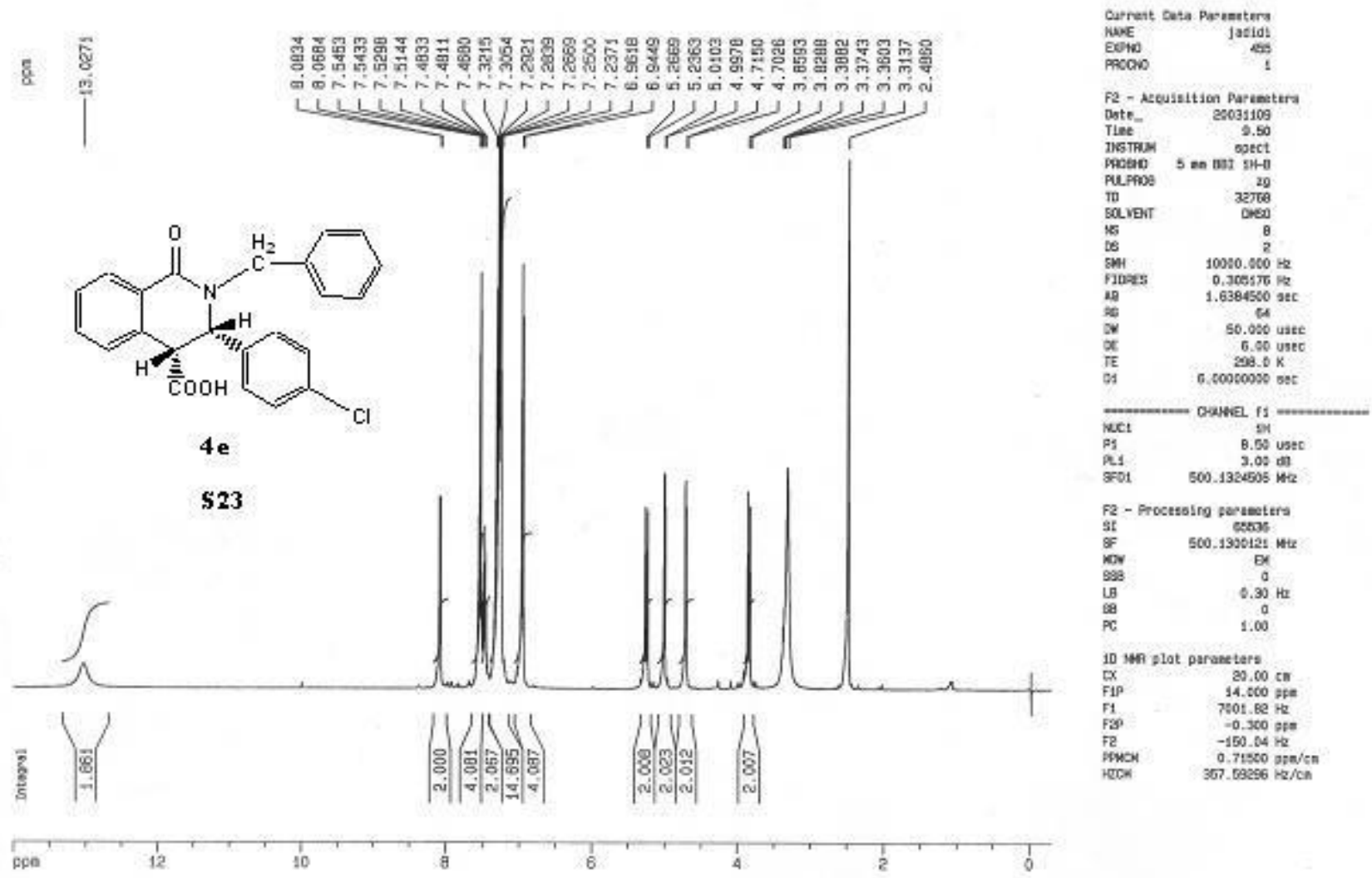


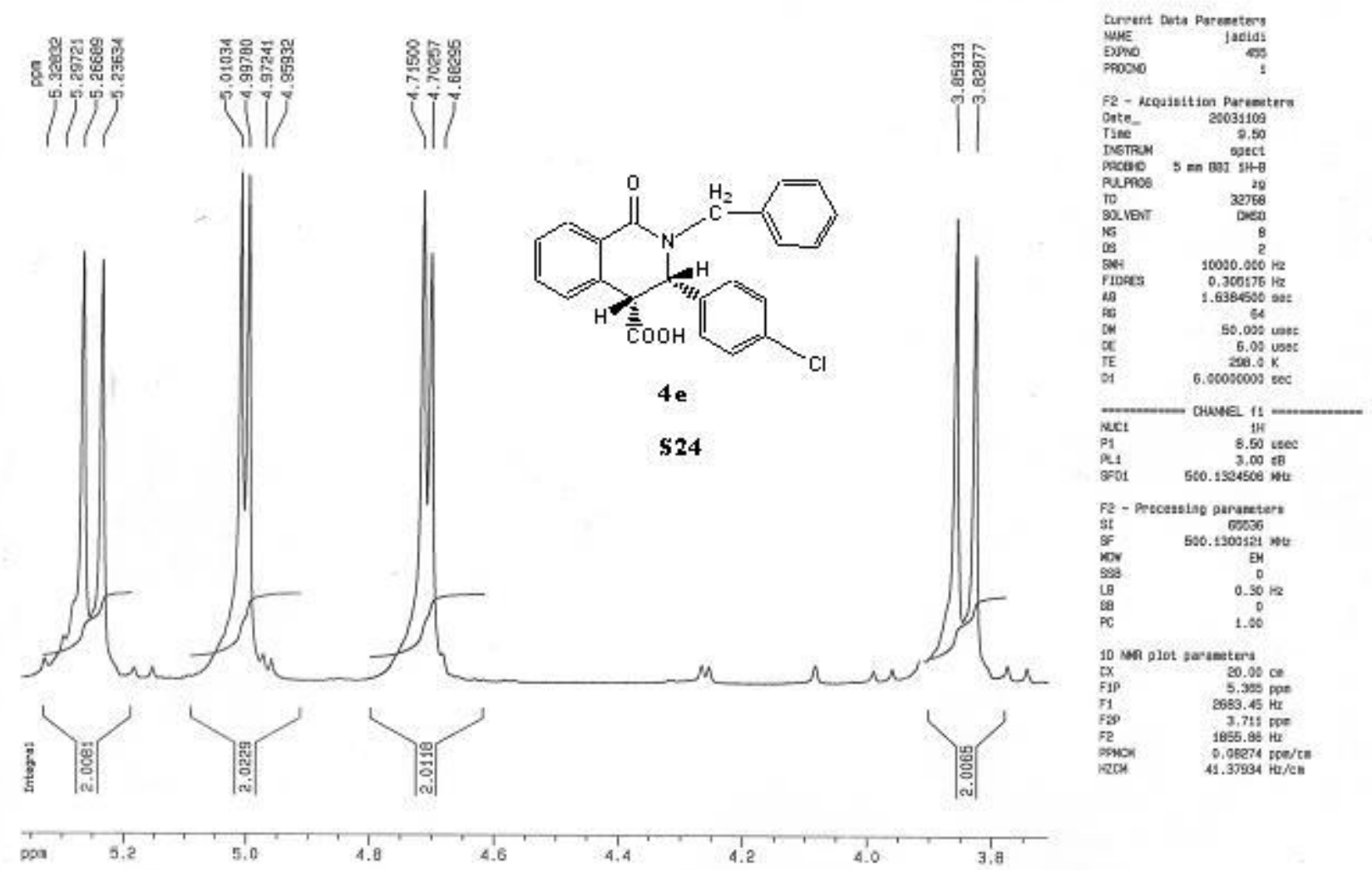




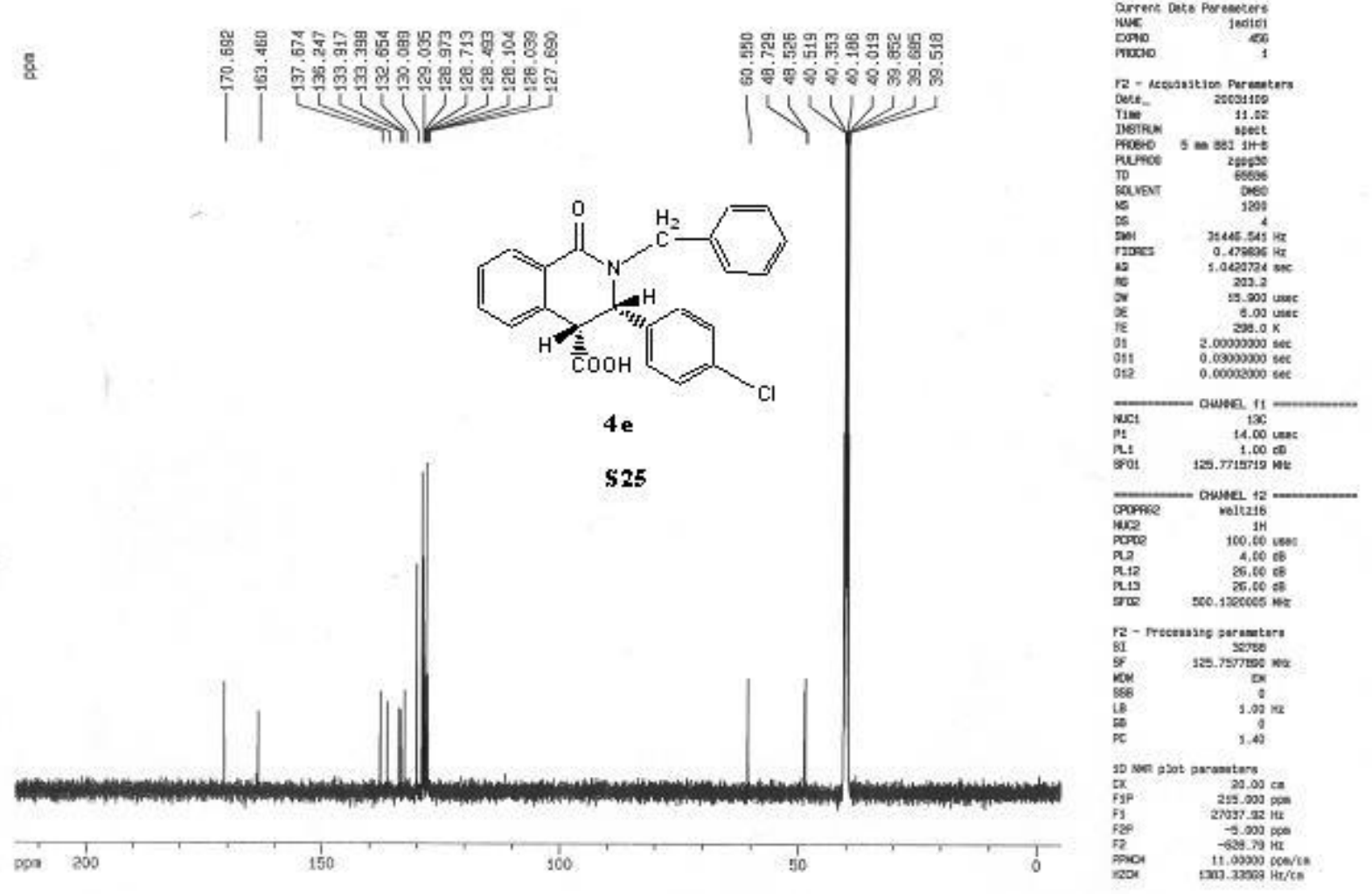


高

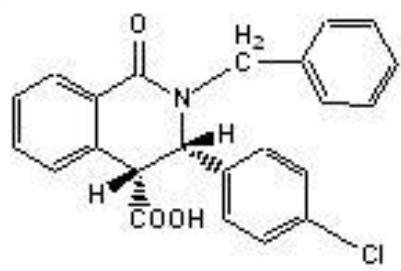

4 e

S26

Whaw 1 .
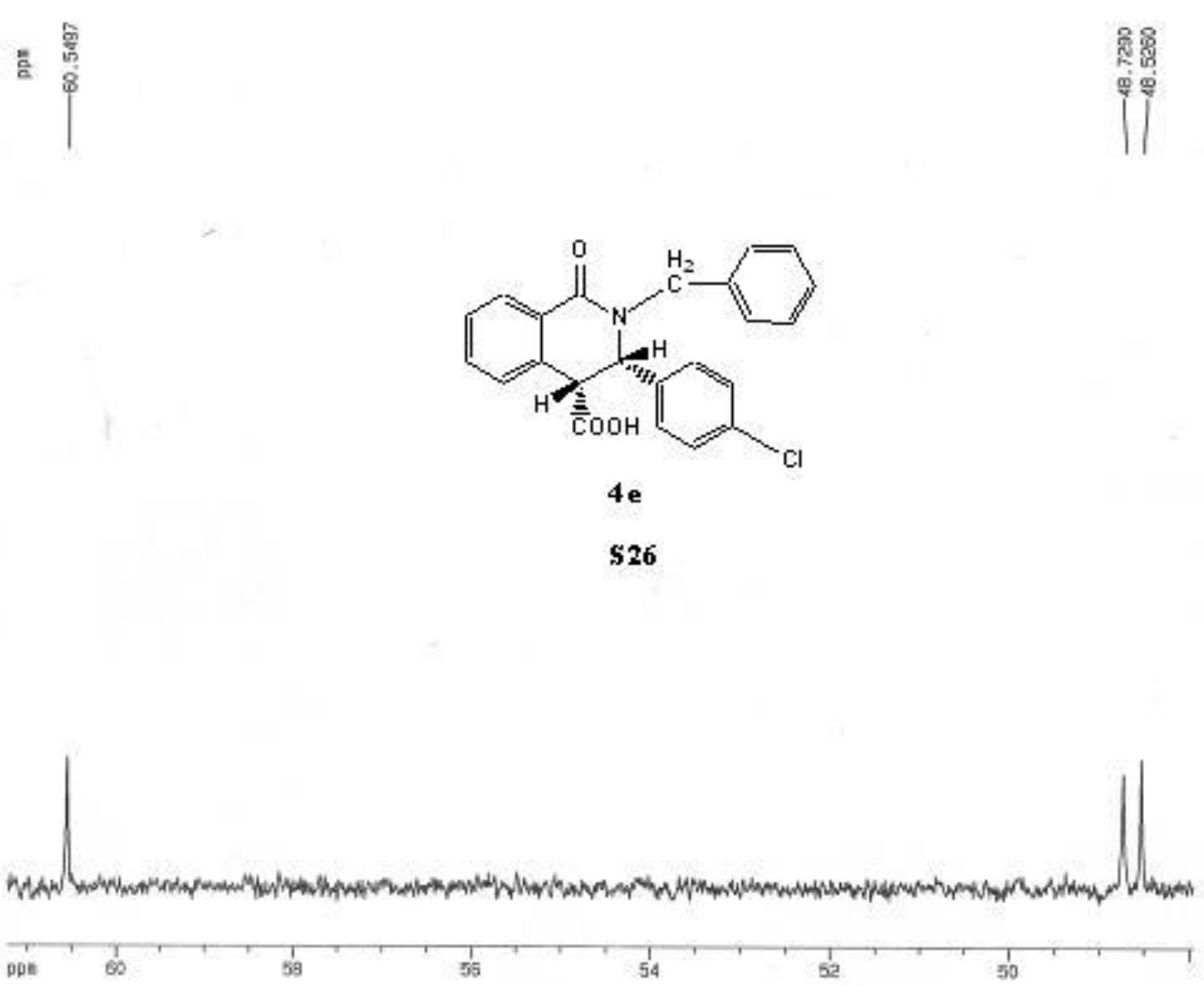

Darrenk Date Pareacters

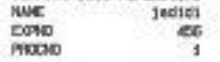

P2 - Nctessitise perianters

Dets- 11 astiss

paober of an sas itte

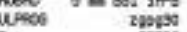

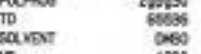

I:445.25s kx

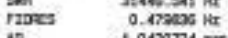

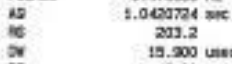

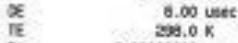

if $\quad 2.0000000 \mathrm{sec}$

a.2 $0.00600000 \mathrm{sec}$

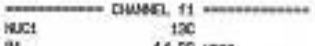

P1. 14.60 taes

ort $1.00 \mathrm{w}$

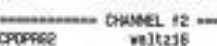

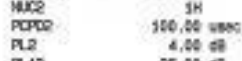

Lit 5,06

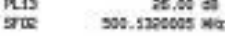

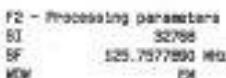

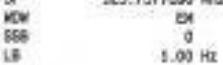

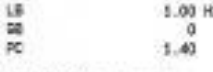

22 iver plot perementara $20.00 \mathrm{~cm}$

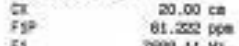

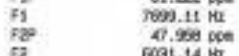

$3004 \quad 6091.14 \mathrm{ke}$ 

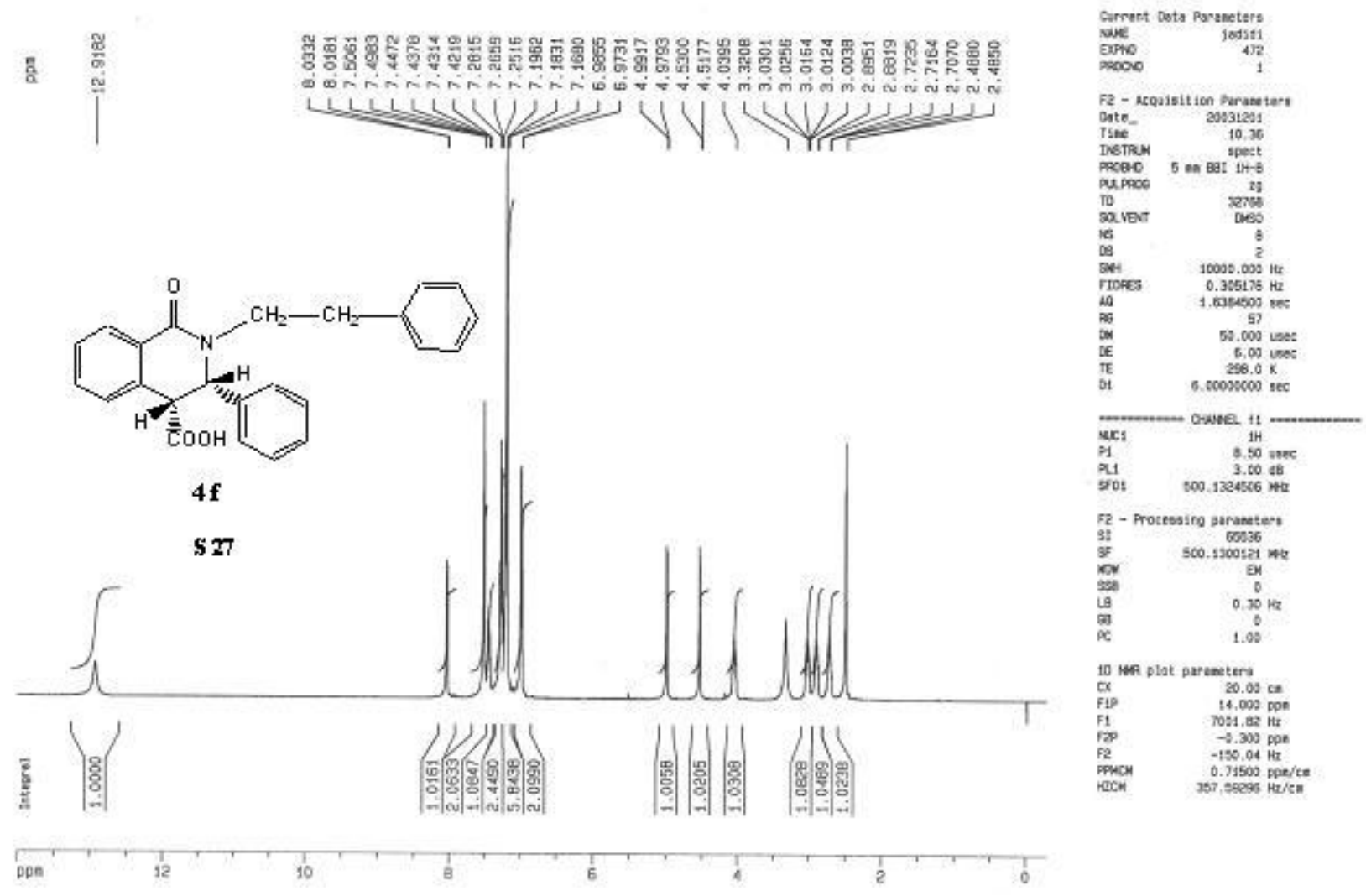

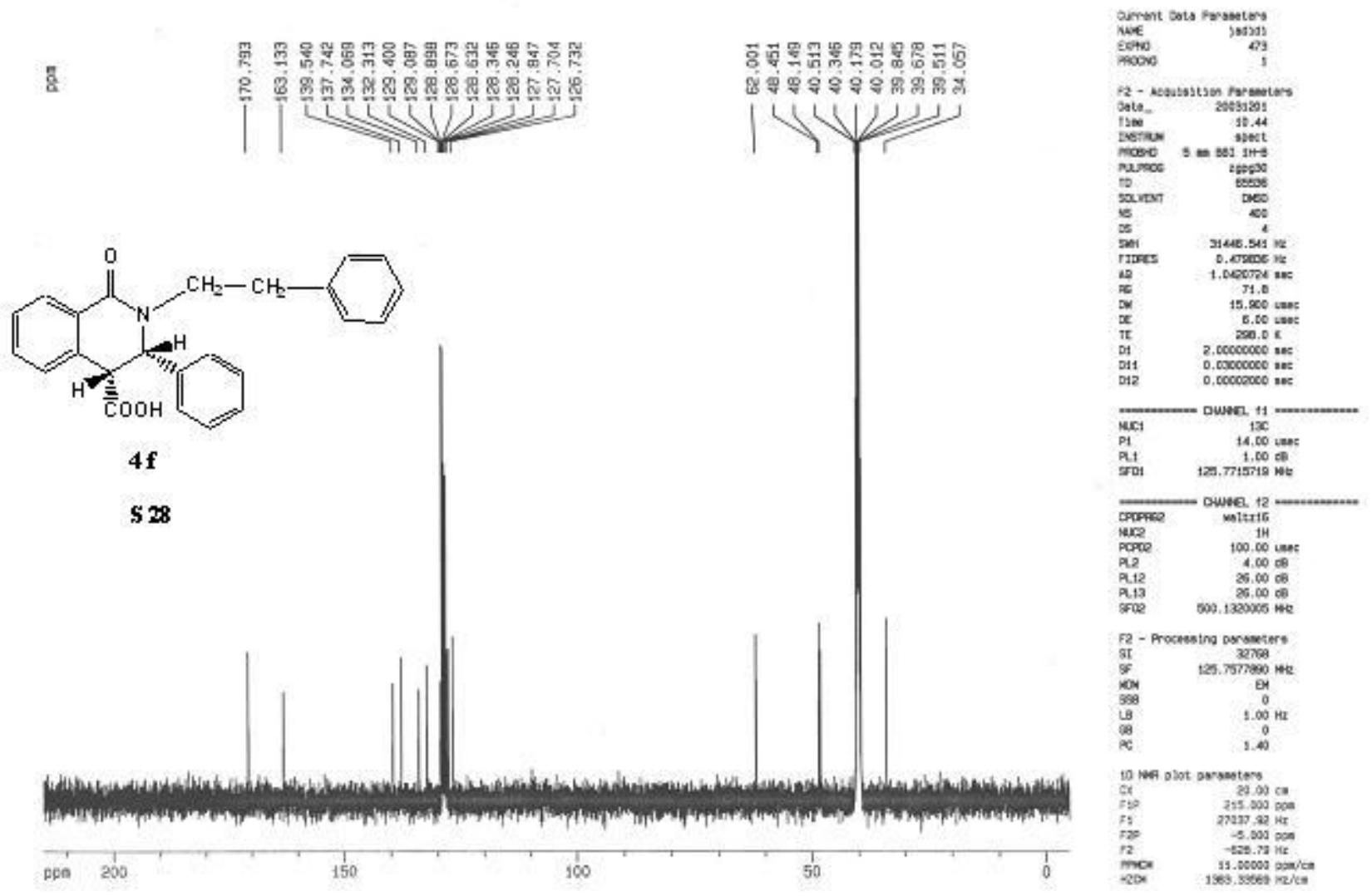


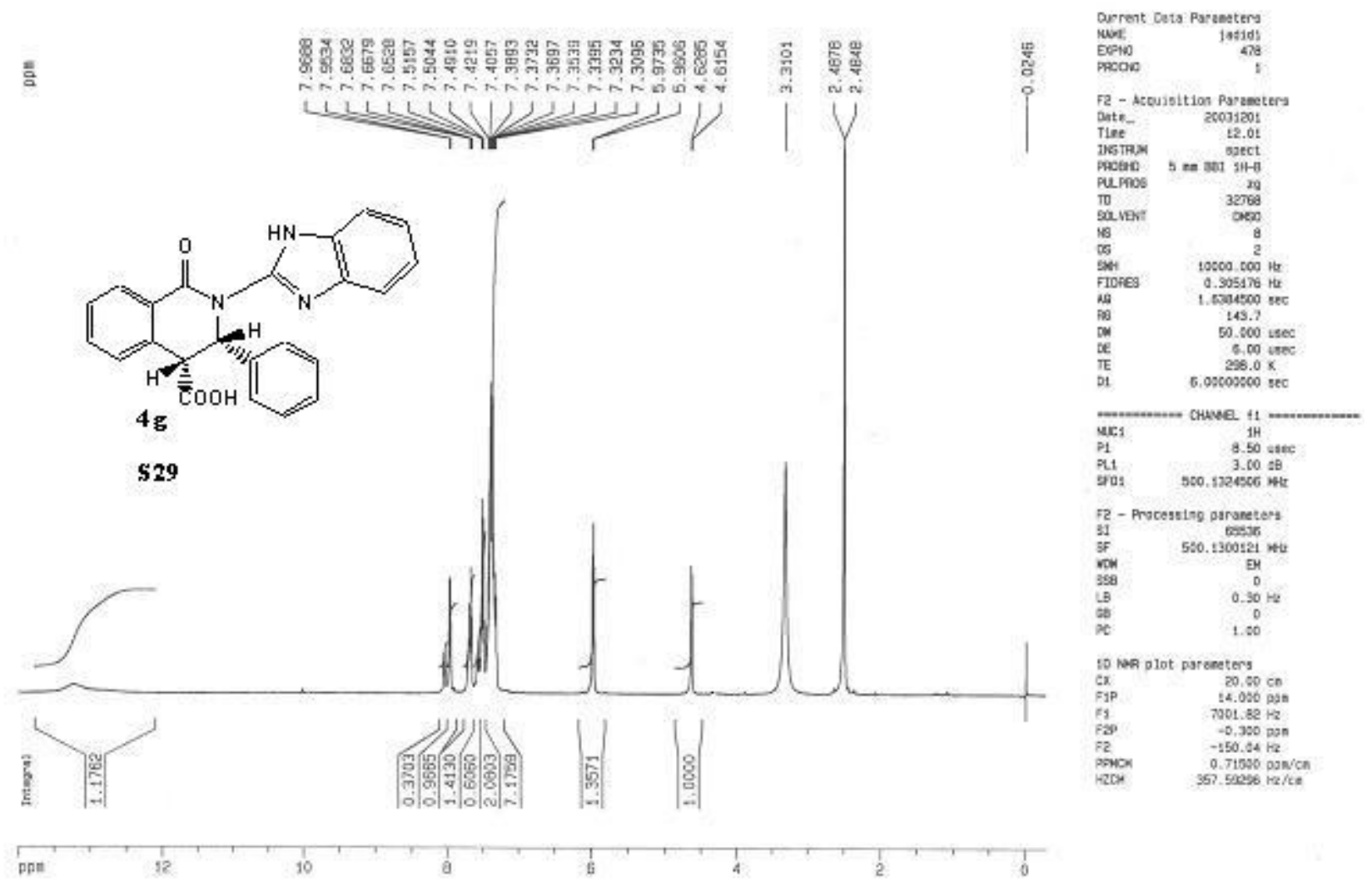




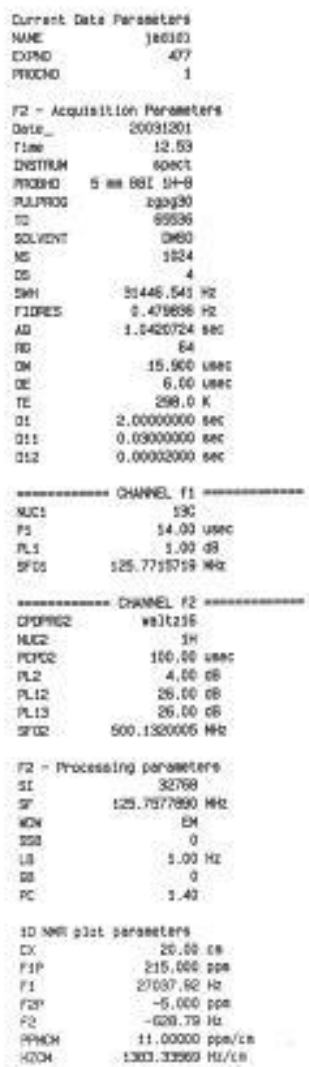



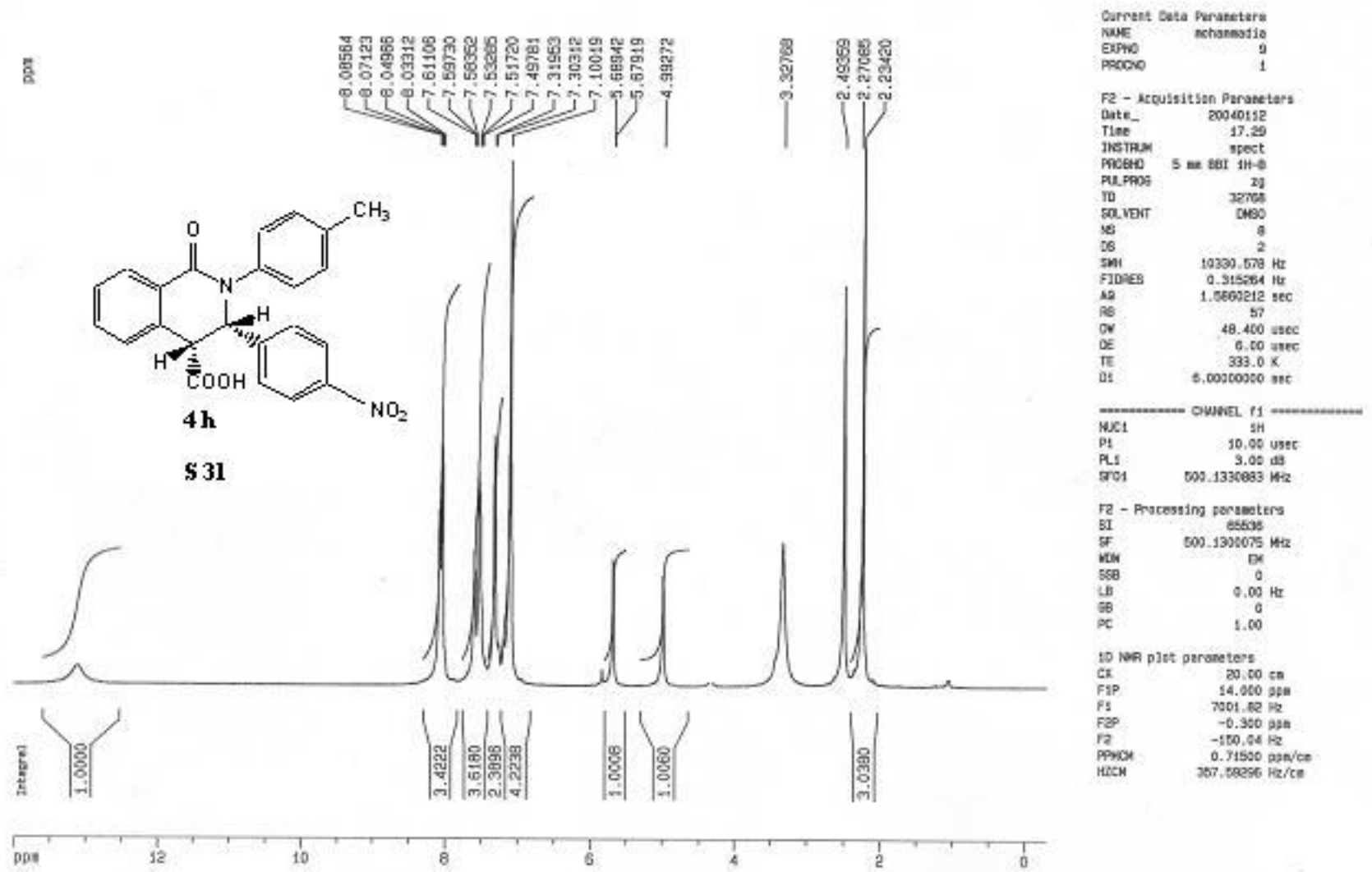

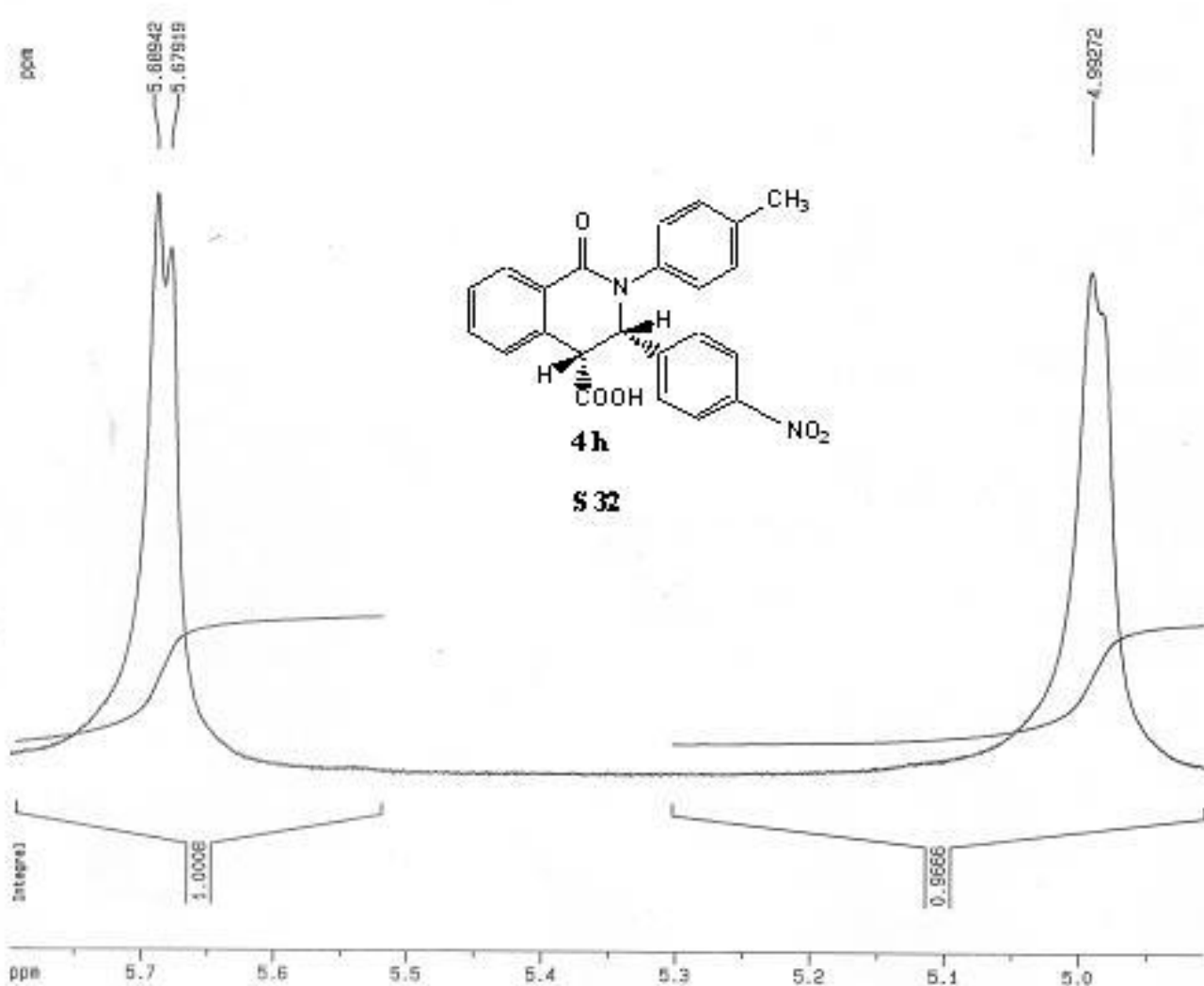

ppe
Cirvent Dots Povenaters

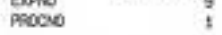

F2 - ucpusettion porimotere

Date_ 205essis

Instram spett

PRCEN $5 \mathrm{mb}$ be: $1 \mathrm{t}+\mathrm{\theta}$

purabe as

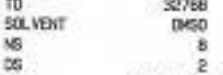

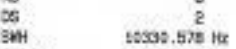

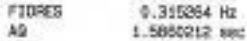

ov 57

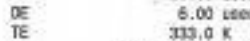

ot 6.00500060 sec

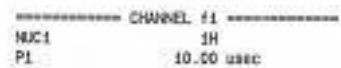

P1 10.60 uat

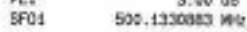

F2 - Praceasima parematers

S1 65536

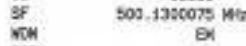

358

Ls $\quad 0.09$

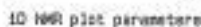

Ge pare peratntars

FD $\quad 5.780 \mathrm{~mm}$

$\begin{array}{ll}\mathrm{Fl} & 2500.10 \mathrm{ke} \\ \mathrm{Fr} & 4.807\end{array}$

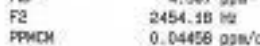

HECM $0.04456 \mathrm{pand} / \mathrm{ch}$ 


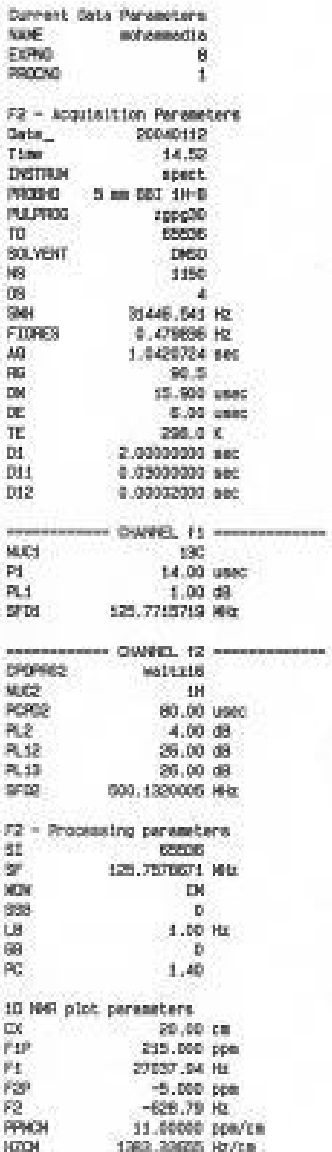<smiles></smiles>

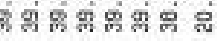

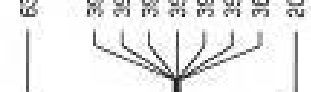

533

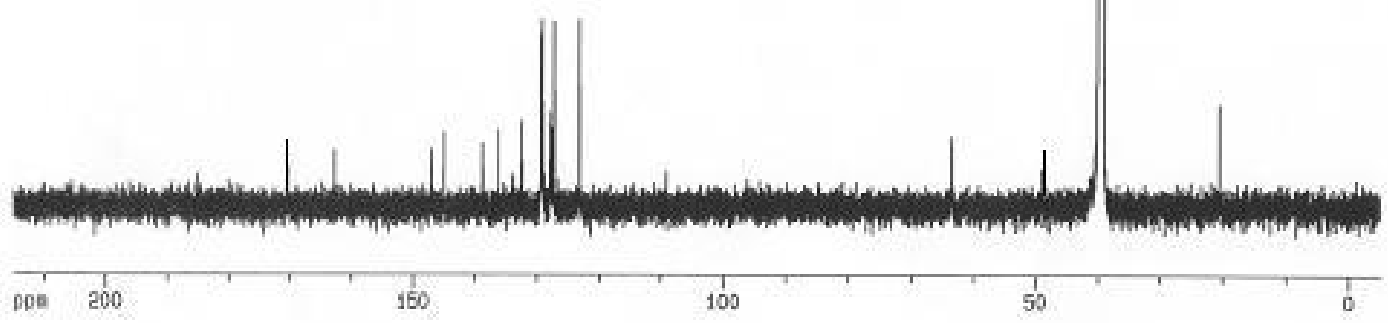



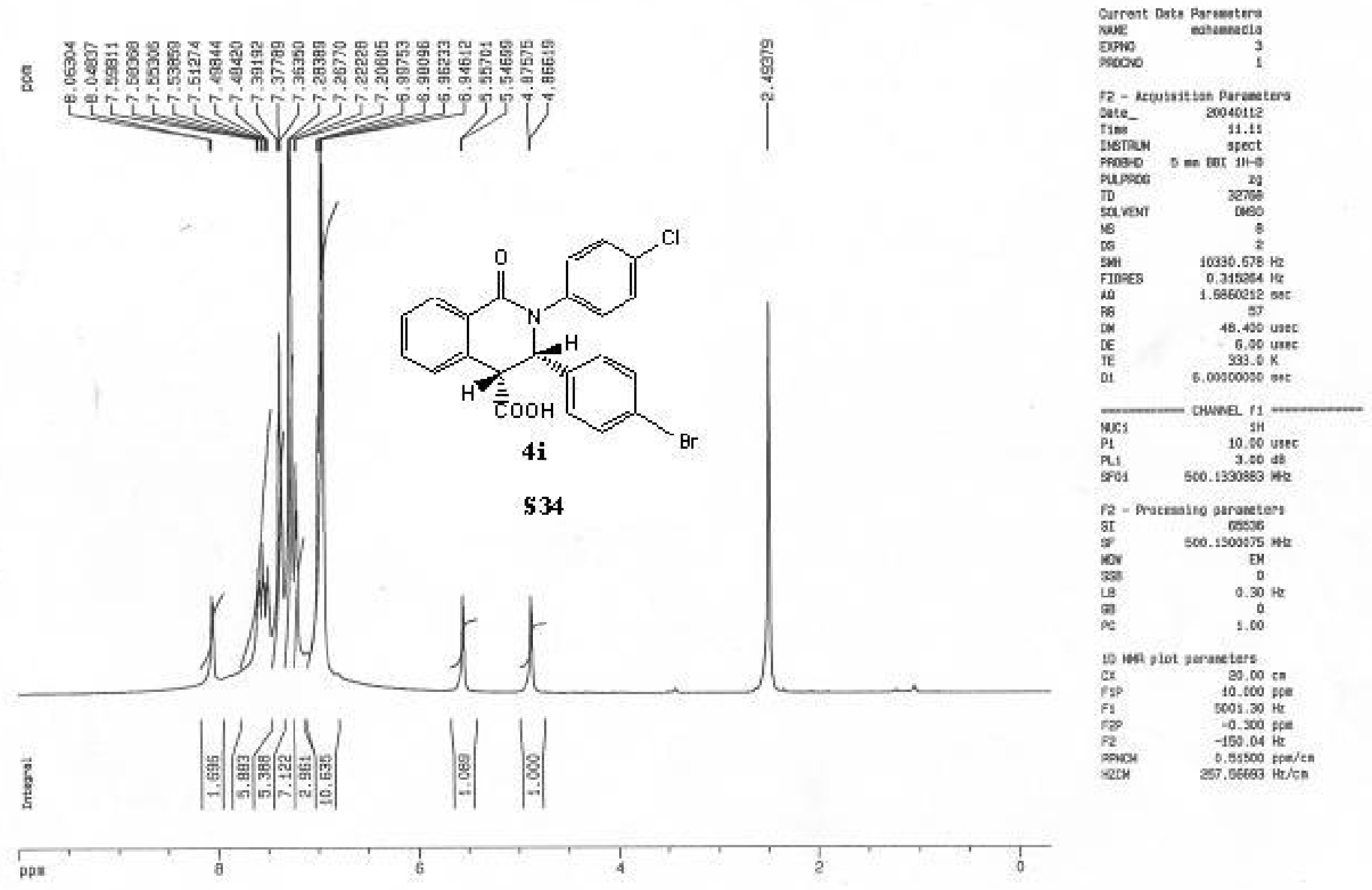


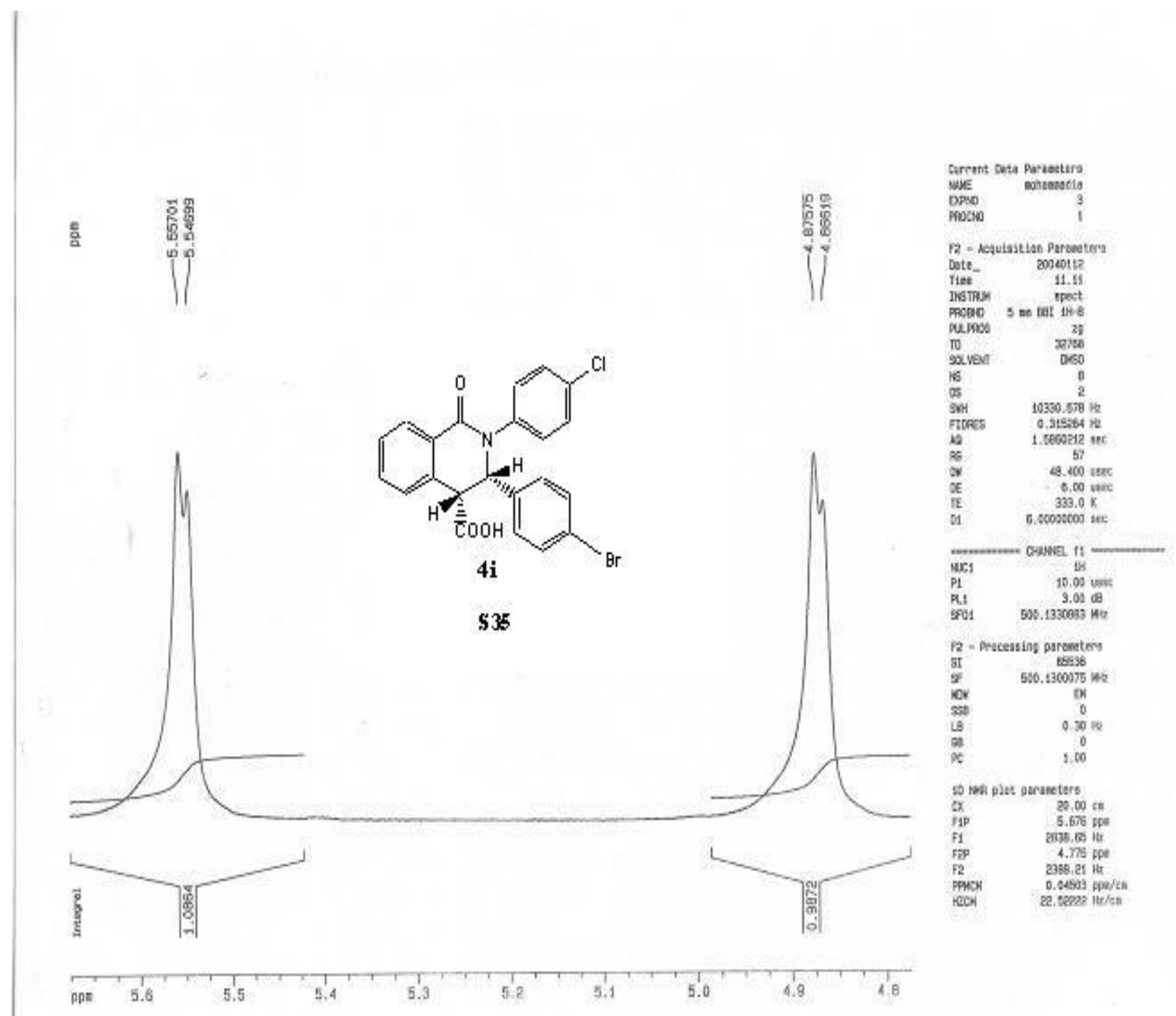




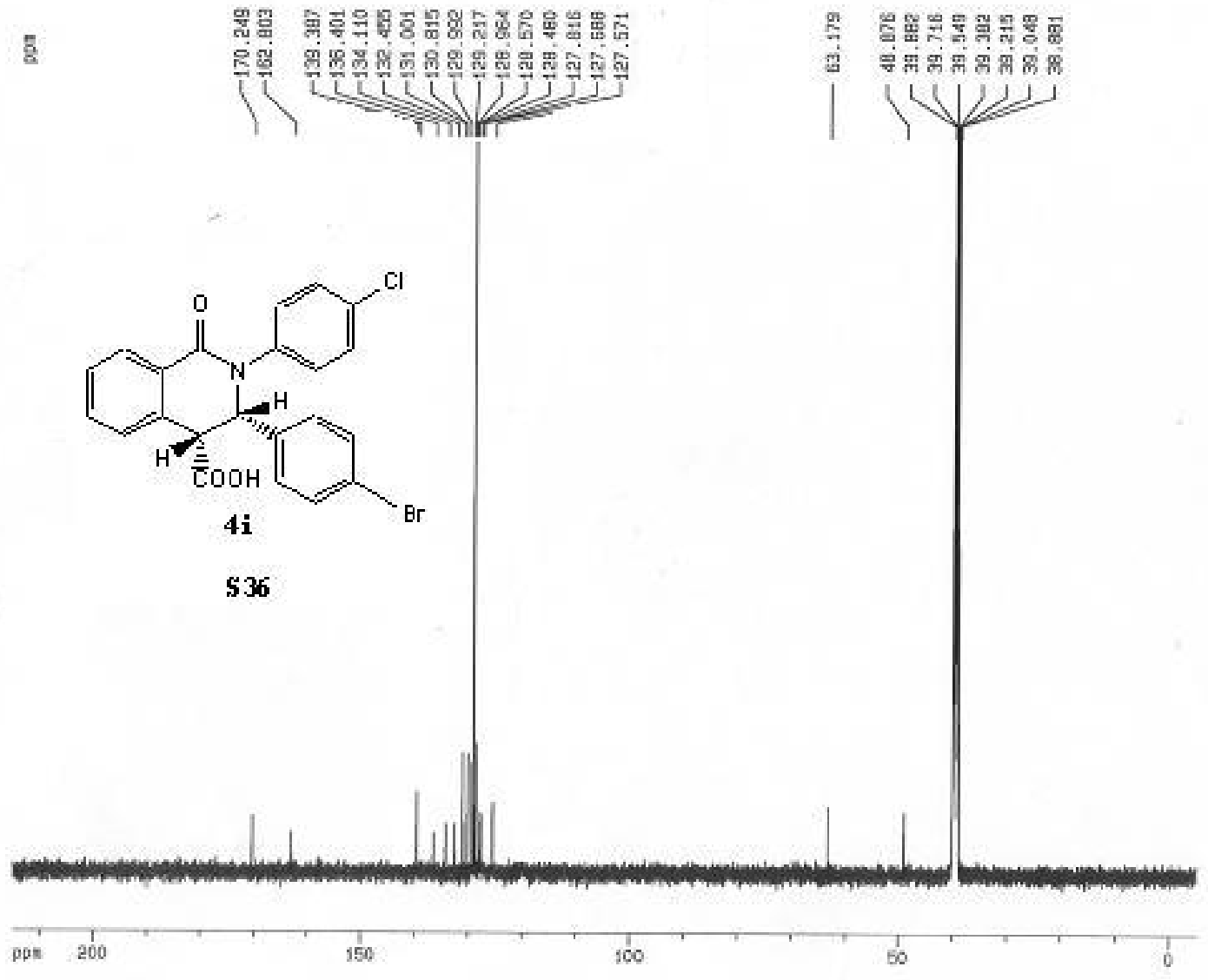



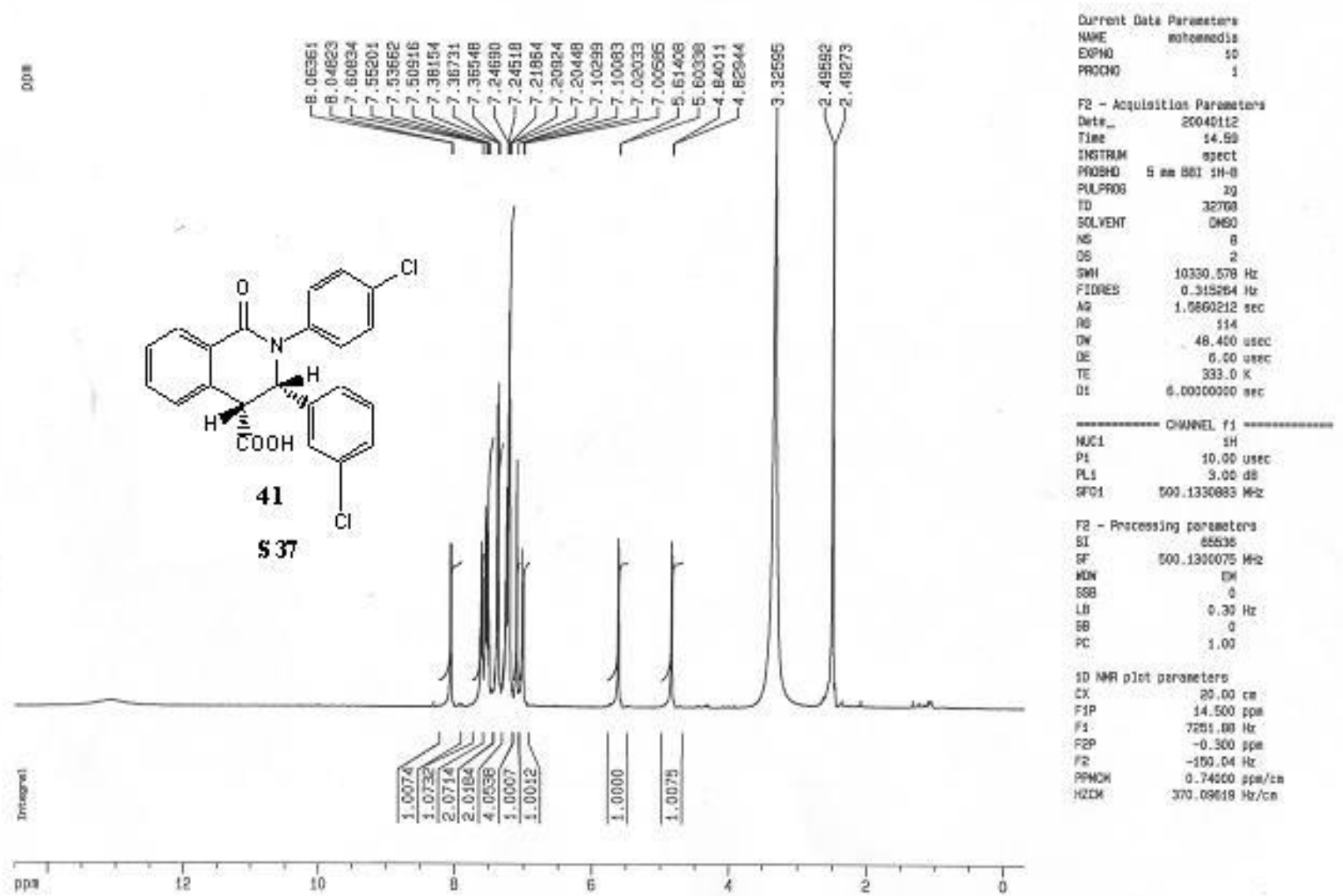


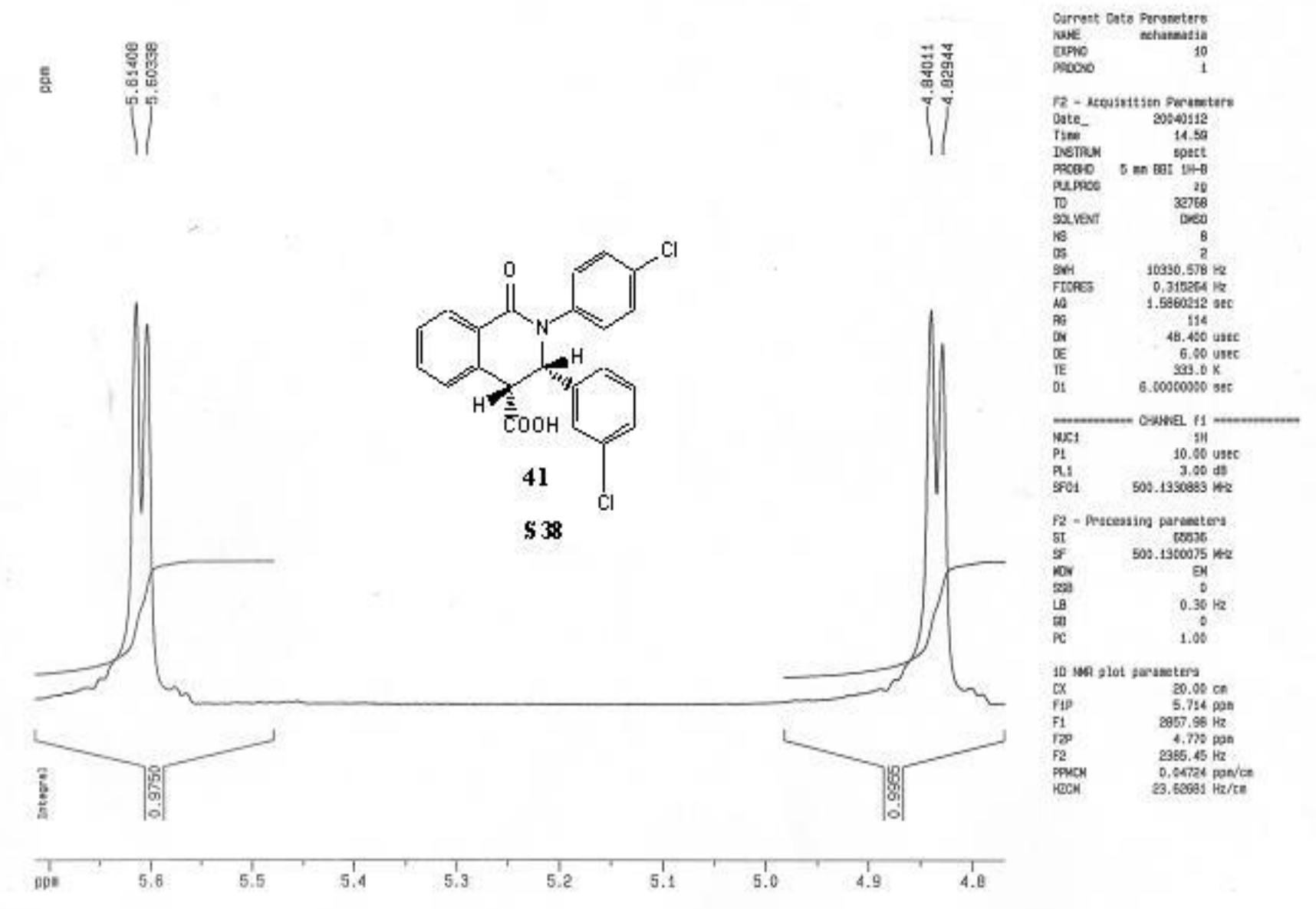




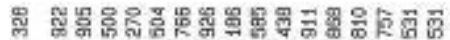

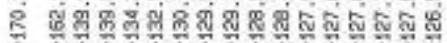

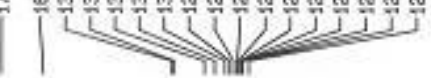

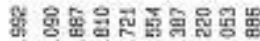

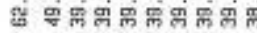

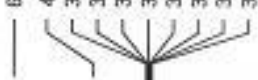

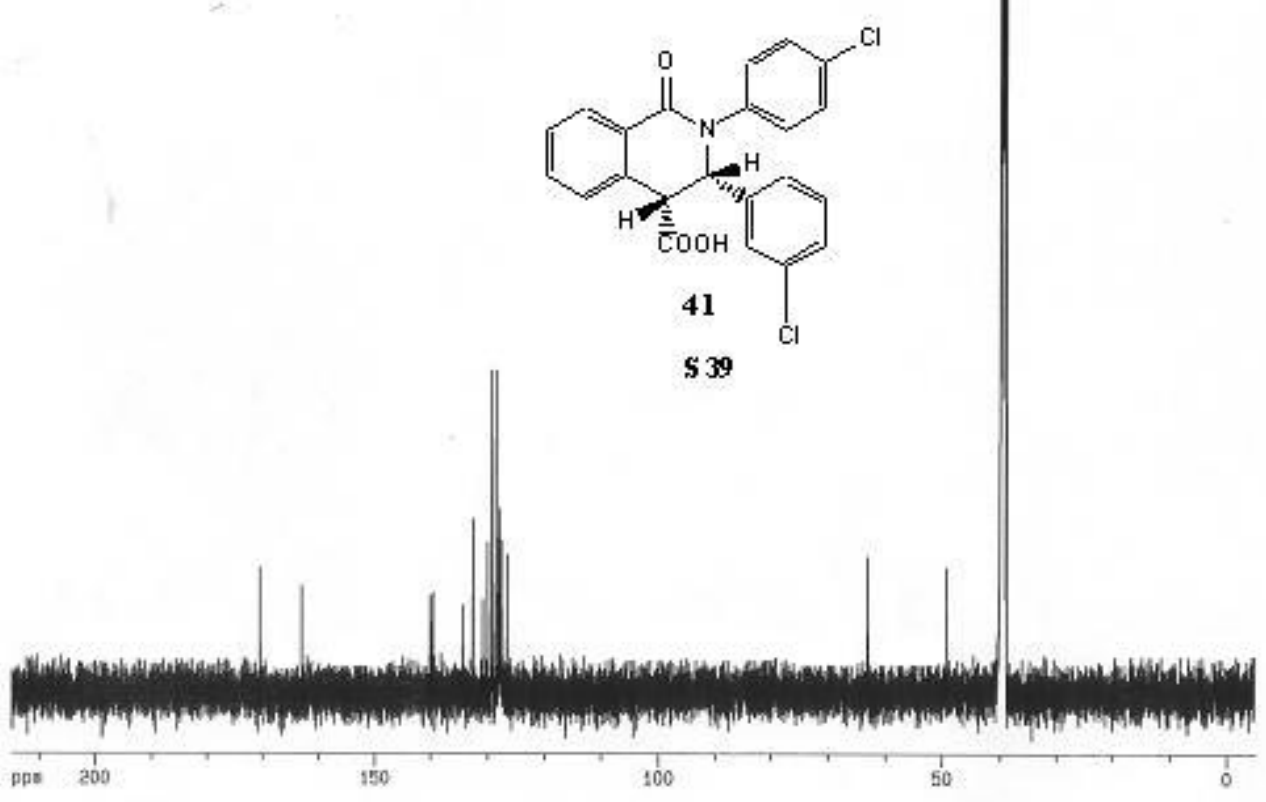



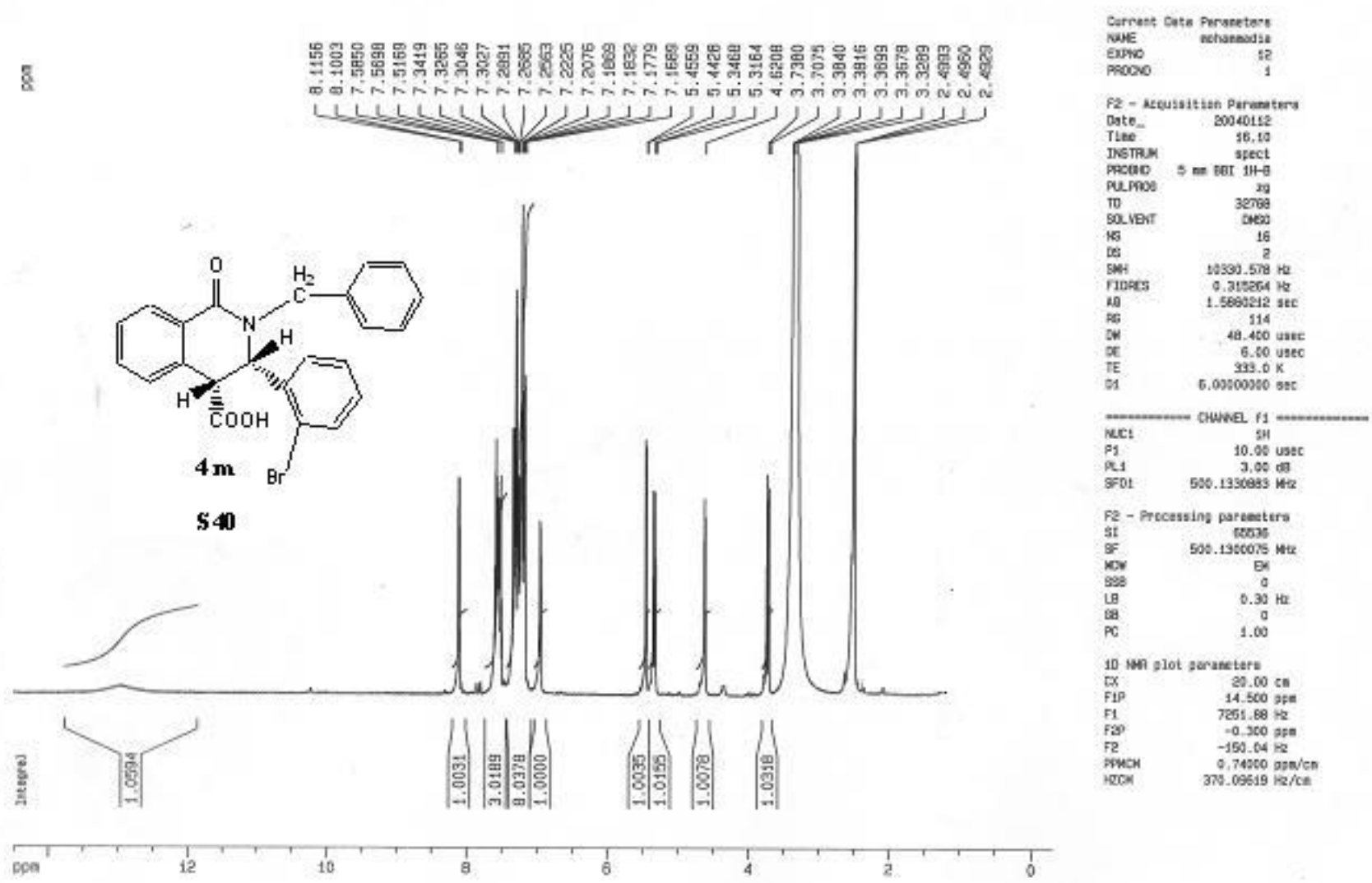


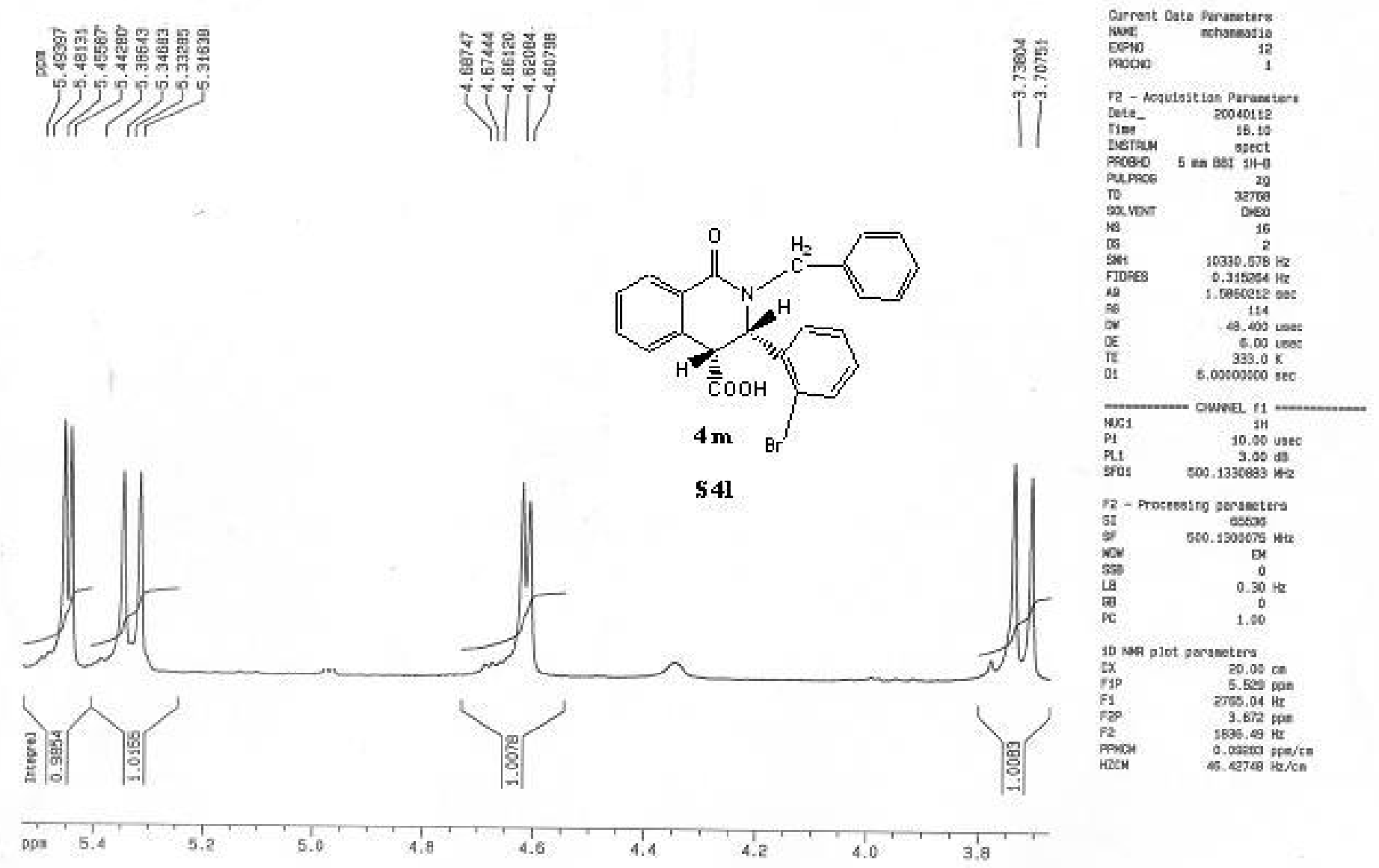


高

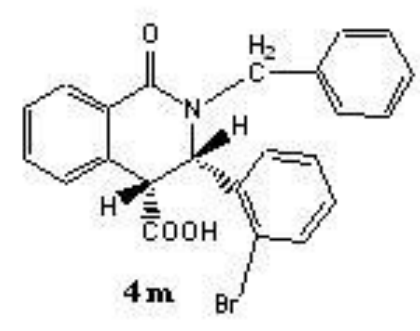

SE

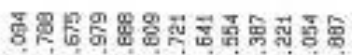

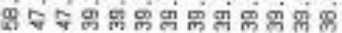

영

int

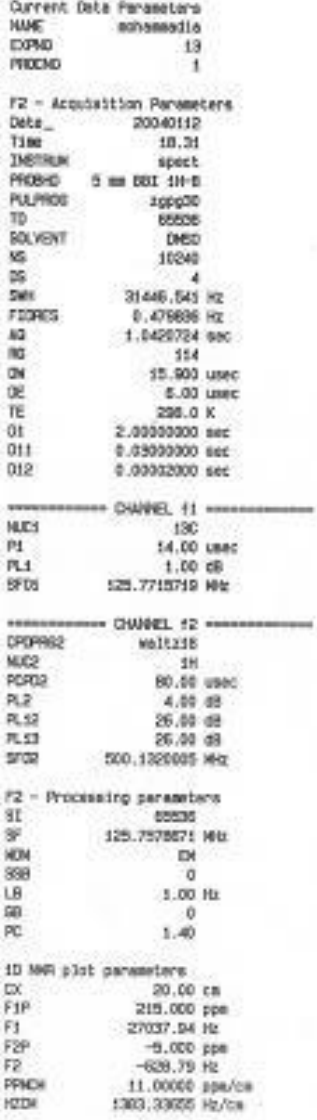

150 
홍
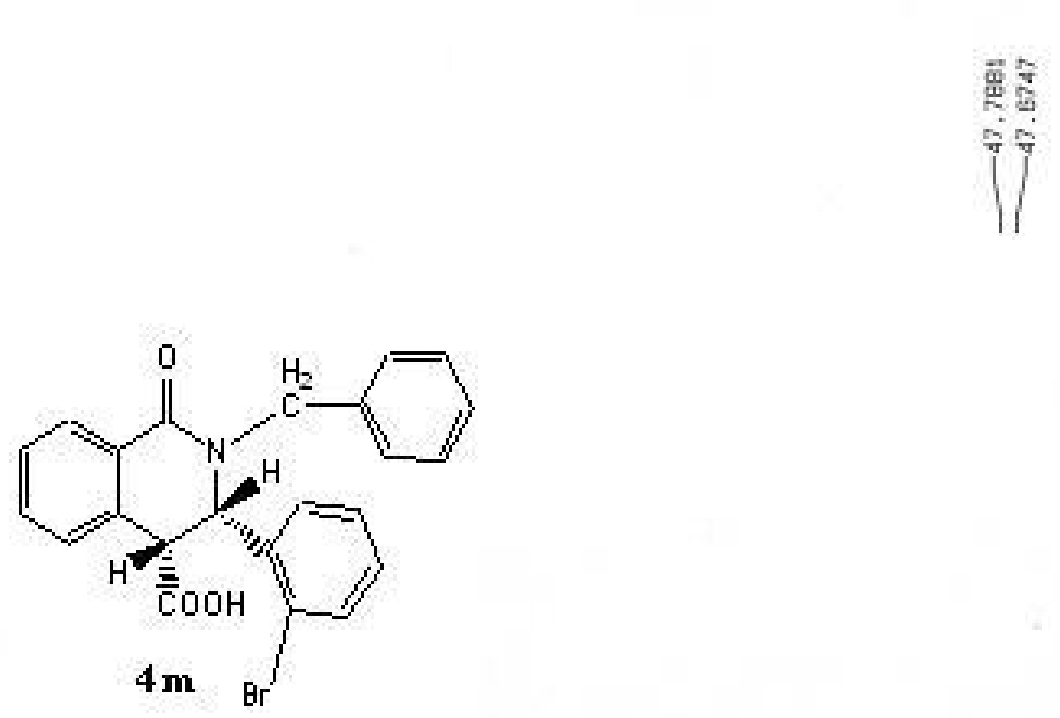

543
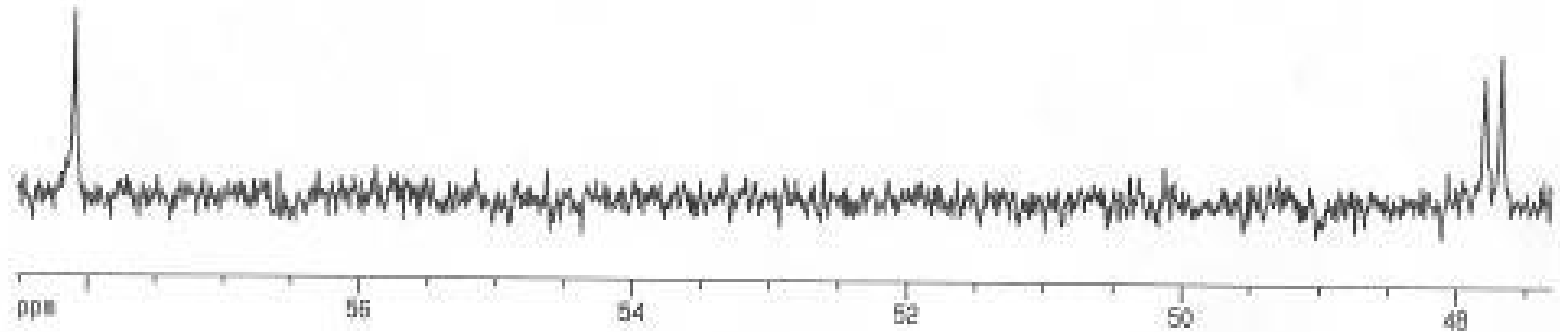

\begin{tabular}{|c|c|}
\hline \multicolumn{2}{|c|}{ Oarwat Oata Prvasstors } \\
\hline E09\% & $\begin{array}{r}\text { monamestis } \\
13\end{array}$ \\
\hline $90 \times 30$ & 1 \\
\hline \multicolumn{2}{|c|}{ F2 - Acquisition Parinentar1 } \\
\hline Date_ & stomise \\
\hline Tine & $18.9 \mathrm{~A}$ \\
\hline netrum & scest \\
\hline phoso & $5=\mathrm{BEI} \mathrm{IH}$ \\
\hline puscce & $2 \sec 30$ \\
\hline TD & 6203 \\
\hline sarent & D69 \\
\hline 8 & seces \\
\hline$\approx$ & 4 \\
\hline 5et. & 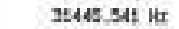 \\
\hline Fones & $0.47 m a g$ its \\
\hline 19 & $1.0400324 \mathrm{met}$ \\
\hline 80 & us \\
\hline 에 & $15.900 \mathrm{Lan}$ \\
\hline $\boldsymbol{\alpha}$ & $6.60 \mathrm{tat}$ \\
\hline$\pi$ & $200.0 \mathrm{~K}$ \\
\hline DA & 2,50000060 ie: \\
\hline bat & $0, \operatorname{cocccces} \mathrm{sec}$ \\
\hline bus & D. eucceeses is \\
\hline \multicolumn{2}{|c|}{ 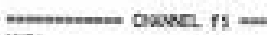 } \\
\hline Ke: & $1 x$ \\
\hline$F$ & 24.02 ung \\
\hline R: & $5.00 \mathrm{do}$ \\
\hline 204 & 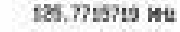 \\
\hline \multirow{2}{*}{\multicolumn{2}{|c|}{ arumes owwe, $12=$}} \\
\hline & voltut6 \\
\hline wes & IH \\
\hline pore & 00.40 LAP \\
\hline A.2 & A.CO $\mathrm{el}$ \\
\hline fute & $26.00 \leqslant 0$ \\
\hline Pus & $20.00 \mathrm{st}$ \\
\hline sfle & $500.135 x c e s$ wot \\
\hline \multicolumn{2}{|c|}{ F2 - Arecossise agrasetars } \\
\hline $\mathrm{sz}$ & 66036 \\
\hline$z$ & 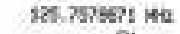 \\
\hline er & or \\
\hline 220 & 0 \\
\hline 18 & t., $00 \mathrm{He}$ \\
\hline 8 & 0 \\
\hline $\boldsymbol{A C}$ & 1,4 \\
\hline \multicolumn{2}{|c|}{ to WA ploh portesters } \\
\hline Dx & $30.00 \mathrm{ts}$ \\
\hline rap & $58.516 \mathrm{sph}$ \\
\hline$r 1$ & $7868.63+\mathrm{iz}$ \\
\hline ras & $0,802,200$ \\
\hline $\mathrm{fe}$ & $2009,80 \times 2$ \\
\hline Pmo: & 0.565150004 \\
\hline IQD & $7.4407 \mathrm{lech}$ \\
\hline
\end{tabular}

